\title{
Steep Progressive Waves in Deep and Shallow Water
}

\author{
Rodney J. Sobey \\ Department of Civil and Environmental Engineering, \\ Imperial College London, \\ London SW7 2AZ, UK \\ Phone: +44 (0)1289 303479, Fax: +44 (0)20 75945991 \\ E-mail: r.j.sobey@imperial.ac.uk
}

\section{Engineering and Computational Mechanics, 165(1), 2012}

25 June 2012

\begin{abstract}
Analytical solutions for steady progressive waves in deep and shallow water are developed and explored. The solution presentations emphasise the significant overlap in their formulations. Each theory is numerically confirmed as correct to fifth order, and details of the solutions are archived in a manner that anticipates application code. Finally, the limits of validity, separate and overlapping, of the respective analytical solutions are extensively examined. It is suggested that $\omega^{2} h / g \approx 0.65$ separates the region of applicability of the shallow and deep water theories.
\end{abstract}

Keywords Analytical theory, cnoidal waves, extreme wave kinematics, limit waves, progressive waves, short waves, Stokes waves.

\section{Introduction}

Analytical solutions for steady progressive waves in deep (Stokes waves) and shallow (cnoidal waves) water are not new. The literature on Stokes waves in particular is extensive and robust.

The literature on cnoidal waves exists but has largely been ignored, perhaps because it involves special functions and integrals that are unfamiliar, and because it is not straightforward to apply. It deserves much wider appreciation and application. One specific objective of this study is the presentation of a new cnoidal wave theory in a manner that will encourage its wide application in practice. This is pursued in a manner that largely 
parallels the development of Stokes wave theory. In achieving this objective, a new Stokes theory has also been developed.

The final objective is to pursue the limits of validity of both Stokes and cnoidal wave theory. One aspect is their continuing validity on approach to the limit wave. A second aspect is their region of overlap, the shallow water limit of Stokes waves and the deep water limit of cnoidal waves.

The initial discussion summarises the common features of analytical theories for steady progressive waves. The rationale for distinct deep and shallow water theories is related to the conflicting length scales of deep and shallow water waves. The common physical basis is emphasised in the discussion of the mathematical formulation and the dispersion relationship. A new deep water (Stokes) theory is introduced. It is confirmed as correct to fifth order and its range of validity is extensively explored. Subsequently, a new shallow water (cnoidal) theory is introduced, in a manner that parallels as closely as possible the simpler deep water theory. This theory also is confirmed as correct to fifth order and its range of validity is extensively explored. Finally, attention is directed to the region of overlapping validity, leading to recommendations for regions of applicability of each theory.

\section{Length Scales in Steady Wave Theory}

Steady wave theory addresses a uniform long-crested wave train propagating on a steady, uniform current in incompressible water of constant depth. The horizontal bed is rigid and impermeable and any current is collinear with the direction of propagation of the wave train.

As for many complicated problems in fluid mechanics, dimensional analysis provides some useful insights. There are three identifiable length scales in a steady wave problem, namely the water depth $h$, the wave height $H$ and the wavelength $L$.

Casual observation of deep water waves would suggest the wavelength as the dominant length scale, which prompts a dimensionless representation as

$$
f\left(\frac{h}{L}, \frac{H}{L}\right)
$$

This grouping of $h / L$ and $H / L$ is essentially the motivation for the Stokes' approximation to nonlinear waves. Deep water waves are characterised by $h / L$ values of order one, i.e. $h / L=O(1)$. Predictive equations in this approximation are strongly dependent on the depth parameter $h / L$, and moderately on the wave height or wave steepness parameter $H / L$. The linear wave theory belongs to this family, in the limit of small wave steepness.

Similar casual observation of shallow water waves suggests the water depth as the dominant length scale, which prompts a dimensionless representation as

$$
f\left(\frac{L}{h}, \frac{H}{h}\right)
$$




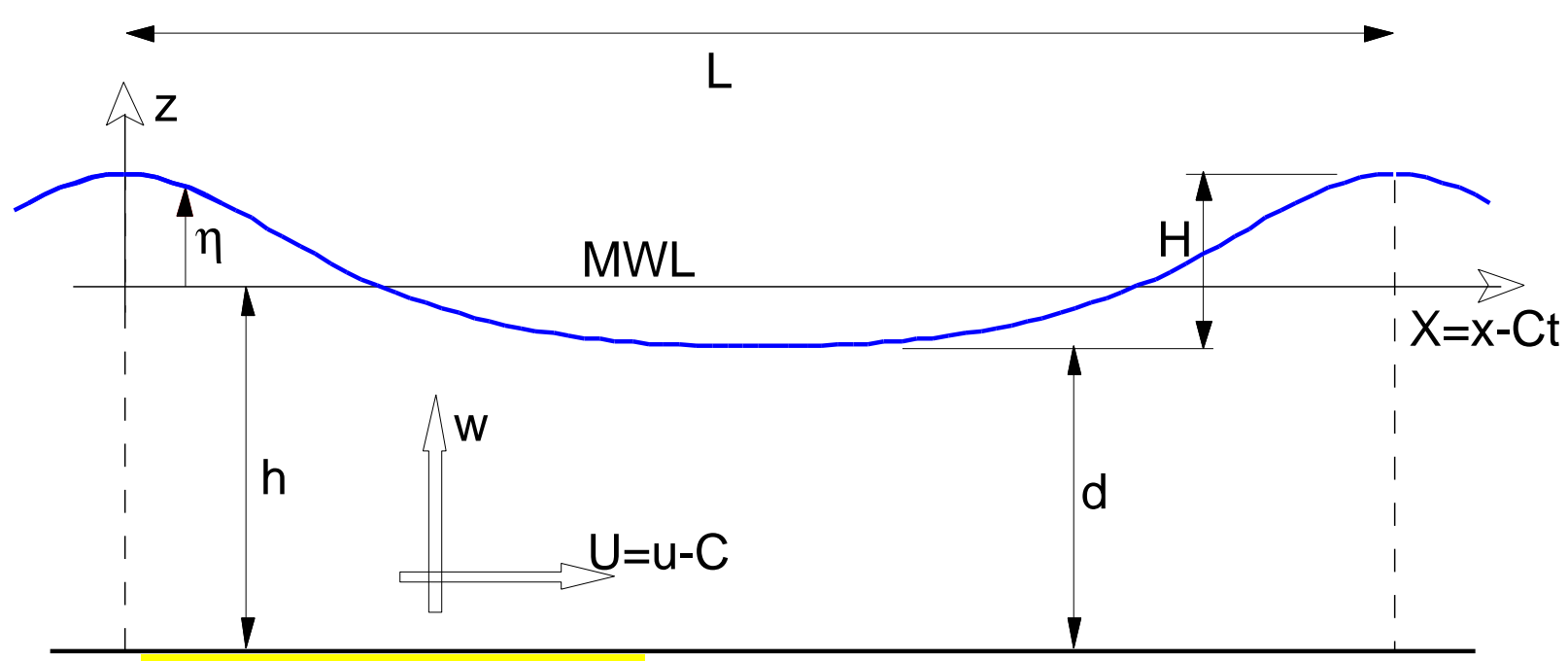

Figure 1: Definition sketch for steady progressive wave.

This grouping of $L / h$ and $H / h$ is the implicit motivation for the cnoidal approximation to nonlinear waves. Shallow water waves are characterised by large values of $L / h$, i.e. $L / h \gg 1$. The predictive equations in this approximation are again strongly dependent on the depth parameter $L / h$, and moderately on the wave height parameter $H / h$.

There is of course an overlapping region where all three length scales may be similarly influential. There is further attention to this overlapping region in Section 7.

\section{Mathematical Formulation of Steady Wave Theory}

Progressive waves of permanent form are steady in a frame of reference moving with the crest at the phase speed $C$. Accordingly, it is convenient to adopt a moving $(X=x-C t, z)$ reference frame (see Figure 1) that is located at the mean water level (MWL) and that moves at speed $C$ with the wave crest. In this $(X, z)$ frame, the flow is steady, whereas in the fixed $(x, z, t)$ reference frame, the flow is unsteady. Homogeneous, incompressible and irrotational flow is assumed.

The mathematical formulation may be presented in terms of the Euler equations, the velocity potential function, the stream function or the complex potential function. The choice of the stream function $\Psi(X, z)$ in the steady frame has some minor advantage in the representation of the kinematic free surface boundary condition.

In terms of the stream function, the field equation representing mass conservation and irrotational flow is the Laplace equation

$$
\frac{\partial^{2} \Psi}{\partial X^{2}}+\frac{\partial^{2} \Psi}{\partial z^{2}}=0
$$

where the velocity components $(U=u-C, w)$ are $(\partial \Psi / \partial z,-\partial \Psi / \partial X)$. 
Momentum conservation throughout the fluid is represented by the irrotational Bernoulli equation

$$
\frac{1}{2}\left(U^{2}+w^{2}\right)+\frac{p}{\rho}+g z=R
$$

where $R$ is the Bernoulli constant in the steady frame.

In terms of the stream function, the complete boundary constraints are as follows:

(1) The bottom boundary condition (BBC), representing no flow through the horizontal bed, is

$$
\Psi(X,-h)=0 \quad \text { or } \quad \Psi(X, z)=0 \quad \text { at } \quad z=-h
$$

(2) The kinematic free-surface boundary condition (KFSBC), representing no flow through the free surface at $\eta(X)$, is

$$
\Psi(X, \eta(X))=-Q \quad \text { or } \quad \Psi(X, z)=-Q \quad \text { at } \quad z=\eta(X)
$$

where $-Q$ is the constant volume flow rate per unit width under the steady wave. $Q$ is numerically positive and this flow is in the negative $X$ direction. The zero datum for the stream function was established by the BBC.

(3) The dynamic free surface boundary condition (DFSBC), representing constant atmospheric pressure on the free surface, is

$$
\frac{1}{2}\left(U^{2}+w^{2}\right)+g \eta=R \quad \text { at } \quad z=\eta(X)
$$

which follows directly from the irrotational Bernoulli equation in the steady frame.

(4) The wave is periodic. The periodic lateral boundary conditions (PLBC) are

$$
\Psi(X+L, z)=\Psi(X, z), \quad \eta(X+L)=\eta(X)
$$

where $L$ is the wave length.

(5) The wave height is defined as

$$
H=\eta(0)-\eta(L / 2)
$$

(6) Mass conservation requires an invariant mean water level (MWL) such that

$$
\int_{0}^{L / 2} \eta(X) d X=0
$$

In principle, the unknowns of a steady wave solution will be the stream function $\Psi(X, z)$, the water surface profile $\eta(X)$, the wave length $L$, the current or mean fluid speed $-\bar{U}$ at any elevation that is always submerged below the trough, the volume flux $-Q$ and the Bernoulli constant $R$. In specific nonlinear wave theories, the stream function, the water surface profile and the wavelength are represented in terms of other parameters, which take their place as unknowns in the theory.

The given parameters defining a steady wave solution are generally the wave height $H$, the water depth $h$, the wave period $T(=2 \pi / \omega)$ and either the co-flowing Eulerian current $U_{E}$ or the wave-averaged mass transport velocity or Stokes drift $U_{S}$. 


\section{Dispersion Relationship}

Steady wave theory seeks to predict the flow in the moving and steady flow reference frame. In this frame, it will predict both the current $-\bar{U}$ and the volume flow rate $-Q$ under the steady wave, respectively

$$
-\bar{U}=\frac{1}{L} \int_{0}^{L} U(X, z) d X, \quad-Q=\int_{-h}^{\eta(X)} U(X, z) d z
$$

In the $\bar{U}$ definition, the elevation $z$ can be any elevation at or below the trough; $z=-h$ is often convenient. In the $Q$ definition, the location $X$ can be any location, as the flow is steady.

Steady wave theory does not predict the actual speed $C$ of this frame. $C$ is measured in the fixed and unsteady frame, and is dependent also on background flow conditions.

The current in the steady frame is $-\bar{U}$ and the steady frame is moving at speed $C$, so that the current in the fixed and unsteady frame is $U_{E}=-\bar{U}+C$. Similarly, the depthaveraged mass transport velocity in the steady frame is $-Q / h$, so that the depth-averaged mass transport velocity in the fixed and unsteady frame is $U_{S}=-Q / h+C$.

Transformation between the frames requires knowledge of both the current and the wave period.

(i) Where the Eulerian current $U_{E}$ is known, the phase speed is

$$
C=\bar{U}+U_{E}
$$

The depth-averaged mass transport velocity or Stokes drift $U_{S}$ is then available from

$$
C=\frac{Q}{h}+U_{S}
$$

(ii) Where the depth-averaged mass transport velocity $U_{S}$ is known, Eq. 13 predicts the phase speed. Eq. 12 then predicts the Eulerian current.

These alternative coordinate transformations are often called Stokes' first and second "definitions" of phase speed.

In either case, the space and time periodicity of the progressive wave of permanent form requires

$$
C=\frac{L}{T}
$$

as a simultaneous equation. The wave period $T$ is the period measured in a fixed reference frame, e.g., by a wave staff or buoy at a fixed position. Similarly, $U_{E}$ is the time-mean Eulerian current in the fixed reference frame, as measured by a current meter deployed at a fixed position below the wave trough.

Taken together, 
(i) Eqs. 12 and 14 where the Eulerian current is known -

$$
\frac{L}{T}=\bar{U}+U_{E}
$$

(ii) or Eqs. 13 and 14 where the depth-averaged mass transport velocity is known -

$$
\frac{L}{T}=\frac{Q}{h}+U_{S}
$$

relate the space and time periodicity. This is the dispersion relationship for the progressive wave. Whichever equation is not used becomes the definition equation for the other "current." With $L, \bar{U}$ and $Q$ predicted by a steady wave theory, the details will vary with the wave theory. The equation is always implicit, as also for linear theory. It must be solved numerically before transformation of the predictions in the steady frame to the fixed frame.

The Section 2 discussion of length scales included the wave length $L$ along with the water depth $h$ and the wave height $H$. While the wave length $L$ is a convenient choice for rationalising the competing analytical theories, $L$ is part of the solution in the steady reference frame, and not given information characterising a wave field in the fixed and unsteady frame. $L$ is naturally replaced by $g / \omega^{2}$ in the fixed frame, where the wave field may generally be characterised as

$$
\text { Wave Field }=f\left(\frac{\omega^{2} h}{g}, \frac{\omega^{2} H}{g}\right)
$$

This scaling is routinely adopted in the following discussion.

\section{$5 \quad$ Stokes Waves to Fifth Order}

The rationale for the Stokes approximation to nonlinear steady waves is provided by the observation (Eq. 1) that the wavelength $L$ is the dominant length scale in deep water. Water depth and wave height are influential through the dimensionless groupings $h / L$ and $H / L$ respectively, where $h / L$ is understood to be of order one.

Following Stokes (1847; see Stokes (1880)), Nishimura et al. (1977), Fenton (1985) and others, a complete Stokes-style expansion has the form

$$
\Psi(X, z)=-\bar{U}(h+z)+\left(\frac{g}{k^{3}}\right)^{1 / 2} \sum_{i=1}^{N} \varepsilon^{i} \sum_{j=1}^{i} A_{i j} \frac{\sinh j k(h+z)}{\cosh j k h} \cos j k X
$$

for the stream function,

$$
\eta(X)=\frac{1}{k} \sum_{i=1}^{N} \varepsilon^{i} \sum_{j=1}^{i} B_{i j} \cos j k X
$$


for the water surface elevation, and

$$
\begin{gathered}
R=\frac{g}{k}\left[C_{0}+\sum_{i=1}^{N} C_{i} \varepsilon^{i}\right] \\
\bar{U}=\left(\frac{g}{k}\right)^{1 / 2}\left[D_{0}+\sum_{i=1}^{N} D_{i} \varepsilon^{i}\right] \\
Q=\left(\frac{g}{k^{3}}\right)^{1 / 2}\left[E_{0}+\sum_{i=1}^{N} E_{i} \varepsilon^{i}\right]
\end{gathered}
$$

for the Bernoulli constant, mean fluid speed and volume flux respectively. The $A, B, C$, $D$ and $E$ are dimensionless coefficients. $N$ is the order of the analytical theory.

Eqs. 18 through 22 are small parameter perturbation expansions in parameter $\varepsilon$. Each has been normalised in terms of $k(=2 \pi / L$ and representing the dominant length scale $L)$ and $g$, and are a variation on those adopted by Fenton (1985). The major differences are the algebraic form for the free surface expansion $\left(\cos j k X\right.$ instead of $\left.\cos ^{j} k X\right)$ and the inclusion of the normalising factor $1 / \cosh j k h$ for the hyperbolic functions. In application, the argument $j k(h+z)$ can become large; the hyperbolic sines and cosines approach $\frac{1}{2} \exp j k(h+$ $z)$, becoming very large and often challenging the resolution of computer software. The hyperbolic function quotients in Eq. 18 remain finite.

The Eq. 18 form for the stream function exactly satisfies the field equation (Eq. 3), the bottom boundary condition (Eq. 5) and the periodic lateral boundary conditions (Eq. 8). The Eq. 19 form for the water surface elevation also exactly satisfies the mean water level constraint (Eq. 10) and the periodic lateral boundary conditions. The dimensionless coefficients $A_{i j}$ through $E_{i}$ remain to be determined by the wave height constraint (Eq. 9) and the free surface boundary conditions (Eqs. 6 and 7).

Of the dimensionless groupings identified in Eq. 1, the primary grouping $h / L$ (or $k h$ ) is implicit in the Eq. 18 representation for the stream function. It is appropriate to adopt the secondary parameter $H / L$ in the definition of the expansion parameter. Following Isobe \& Kraus (1983), the form adopted is

$$
\varepsilon=\frac{k H}{2}
$$

The choice for the expansion parameter is not inconsequential. Stokes definition of $\varepsilon=$ $k B_{11}$ leads (Schwartz, 1974) to a theory that is not convergent for deep water waves beyond a certain height that is less than the maximum. Eq. 23 has been adopted successfully in Stokes-style theories by Isobe \& Kraus (1983), Fenton (1985) and Kishida \& Sobey (1988) for progressive waves, and by Sobey (2009) for standing waves.

At zeroth order $\left(\varepsilon^{0}\right)$, there is no wave, $\eta(X) \equiv 0$, and the steady flow is uniform and critical, so that

$$
R=\frac{1}{2} C^{2}, \quad \bar{U}=C \quad \text { and } Q=\bar{U} h
$$


For Stokes waves at very small wave height, the phase speed $C$ is $(g / k \tanh k h)^{1 / 2}$. Accordingly,

$$
C_{0}=\frac{1}{2} \tanh k h, \quad D_{0}=(\tanh k h)^{1 / 2}, \quad E_{0}=k h(\tanh k h)^{1 / 2}
$$

Hyperbolic function quotients appear routinely in the kinematic and dynamic free surface boundary condition equations, through $\Psi(X, z)$ and also the velocity components

$$
\begin{aligned}
& U(X, z)=\frac{\partial \Psi}{\partial z}=-\bar{U}+\left(\frac{g}{k}\right)^{1 / 2} \sum_{i=1}^{N} \varepsilon^{i} \sum_{j=1}^{i} j A_{i j} \frac{\cosh j k(h+z)}{\cosh j k h} \cos j k X \\
& w(X, z)=-\frac{\partial \Psi}{\partial X}=\left(\frac{g}{k}\right)^{1 / 2} \sum_{i=1}^{N} \varepsilon^{i} \sum_{j=1}^{i} j A_{i j} \frac{\sinh j k(h+z)}{\cosh j k h} \sin j k X
\end{aligned}
$$

The hyperbolic function quotients, evaluated at $z=\eta$ for the free surface boundary conditions, become respectively

$$
\begin{aligned}
& \frac{\cosh j k(h+\eta)}{\cosh j k h}=\cosh (j k \eta)+\tanh (j k h) \sinh (j k \eta) \\
& \frac{\sinh j k(h+\eta)}{\cosh j k h}=\tanh (j k h) \cosh (j k \eta)+\sinh (j k \eta)
\end{aligned}
$$

The $\sinh (j k \eta)$ and $\cosh (j k \eta)$ contributions are accommodated through classical series expansions (equivalently Taylor series expansions about the MWL)

$$
\begin{gathered}
\sinh (j k \eta)=j k \eta+\frac{1}{3 !}(j k \eta)^{3}+\frac{1}{5 !}(j k \eta)^{5}+\ldots \\
\cosh (j k \eta)=1+\frac{1}{2 !}(j k \eta)^{2}+\frac{1}{4 !}(j k \eta)^{4}+\ldots
\end{gathered}
$$

in which $\eta$ is represented as Eq. 19.

Equations 18 through 23, and Equations 25 through 29 are used in

(i) the wave height constraint (Eq. 9),

(ii) the kinematic free surface boundary condition (Eq. 6), and

(iii) the dynamic free surface boundary condition (Eq. 7).

Collecting the coefficients of powers of $\varepsilon$ defines the analytical problem at consecutive orders $1 \ldots N$, which provide sufficient independent algebraic equations to determine the balance of the $A_{i j}$ through $E_{i}$ coefficients.

At each order there are three algebraic coefficient equations, corresponding to constraints (i) through (iii). The free surface boundary condition constraints, (ii) and (iii), naturally contain powers and products of sine and cosine terms at each order above the first, specifically 
(a) at second order: $\sin ^{2}(k X) ; \cos (2 k X)$

(b) at third order: $\cos (k X) \sin ^{2}(k X), \cos (k X) \cos (2 k X), \sin (k X) \sin (2 k X), \cos (3 k X)$

(c) and similar for higher orders.

These power and product terms are expanded as sums of powers of $\cos (k X)$, using standard trigonometric identities. The free surface boundary condition constraints become double power series in $\varepsilon$ and $\cos (k X)$. All terms have the form $\varepsilon^{i} \cos ^{j} k X$ where $i, j=0,1,2,3, \ldots$

As the free surface boundary conditions must be satisfied for all $X$, the coefficients terms must also be zero. At each order, the number of unknown coefficients and the number of independent coefficient equations are balanced, leading to a unique solution and the definition of the dimensionless coefficients $A_{i j}, B_{i j}, C_{i}, D_{i}$ and $E_{i}$. Given the Eq. 25 solution for the zeroth order, all subsequent coefficient equations are linear. Routine use was made of computer algebra software. The resulting coefficients to fifth order are listed in Appendix A. Fifth order, $N=5$, has become a de facto standard for analytical theories, largely as it provides a pragmatic balance between prediction accuracy and increasing complexity of the algebraic equations for the $A_{i j}$ through $E_{i}$ coefficients. It was necessary to complete the solution to sixth order to completely define the $D_{i}$ coefficients to fifth order.

Note the relative simplicity of the coefficient set. $A_{2 j}, B_{2 j}$ and $A_{4 j}, B_{4 j}$ are zero for $j$ odd. $A_{3 j}, B_{3 j}$ and $A_{5 j}, B_{5 j}$ are zero for $j$ even. $C_{i}, D_{i}, E_{i}$ are zero for $i$ odd. The Fenton (1985) coefficients have the same relative simplicity.

Confirmation of Theory. Confirmation of a verifiably correct analytical theory follows (Fenton, 1985) from Richardson extrapolation to the limit. The procedure is based on the kinematic (Equation 6) and dynamic (Equation 7) free surface boundary conditions, reformulated respectively as

$$
F_{K}(X)=\left.\Psi\right|_{X, \eta(X)}+Q, \quad F_{D}(X)=\frac{1}{2}\left[\left.U\right|_{X, \eta(X)} ^{2}+\left.w\right|_{X, \eta(X)} ^{2}\right]+g \eta(X)-R
$$

Both $F_{K}$ and $F_{D}$ should be zero for all $X$, but there will be error. The root-mean-square errors

$$
f_{K}=\left[\frac{2}{L} \int_{0}^{L / 2} F_{K}^{2}(X) d X\right]^{1 / 2}, \quad f_{D}=\left[\frac{2}{L} \int_{0}^{L / 2} F_{D}^{2}(X) d X\right]^{1 / 2}
$$

will be proportional to $\varepsilon^{\mu}$. $\varepsilon$ is the expansion parameter, proportional to the wave height. $\mu$ is a dimensionless exponent that measures the accuracy of the theory. Specifically, $f_{K} \propto \varepsilon^{\mu_{K}}$ and $f_{D} \propto \varepsilon^{\mu_{D}}$. Evaluating the error at two separate wave heights (but the same water depth and wave period) gives separate expansion parameters $\varepsilon_{1}$ and $\varepsilon_{2}$, and separate error estimates $f_{K 1}, f_{K 2}$ and $f_{D 1}, f_{D 2}$. Accordingly

$$
\frac{f_{K 2}}{f_{K 1}}=\left(\frac{\varepsilon_{2}}{\varepsilon_{1}}\right)^{\mu_{K}}, \quad \frac{f_{D 2}}{f_{D 1}}=\left(\frac{\varepsilon_{2}}{\varepsilon_{1}}\right)^{\mu_{D}}
$$


and

$$
\mu_{K}=\frac{\log \left(f_{K 2} / f_{K 1}\right)}{\log \left(\varepsilon_{2} / \varepsilon_{1}\right)}, \quad \mu_{D}=\frac{\log \left(f_{D 2} / f_{D 1}\right)}{\log \left(\varepsilon_{2} / \varepsilon_{1}\right)}
$$

The dimensionless exponents $\mu_{K}$ and $\mu_{D}$ provide independent fidelity measures of an analytical theory coupled with its code implementation. This technique of Richardson extrapolation to the limit deserves a routine place in any application code for higher order waves.

For $h=100 \mathrm{~m}, T=10 \mathrm{~s}, U_{E}=0, H_{1}=0.5 \mathrm{~m}$ and $H_{2}=1.0 \mathrm{~m}, \mu_{K}$ and $\mu_{D}$ are 6.0002 and 5.9999 respectively, confirming that the theory is correct to fifth order.

As an aide to confirmation of implementation code, a complete list of solution coefficients are given in Appendix B for a specific listed wave condition.

Dispersion. The penultimate step in application of the Stokes fifth order theory is the solution of the dispersion relationship, Eqs. 15 or 16 for $k$. In a common application, $h, H$ and $T$ are known.

(i) If the current specified is the Eulerian current $U_{E}$, then Eq. 15 becomes the implicit algebraic equation

$$
f(k)=0=\frac{L(k)}{T}-\bar{U}(k)-U_{E}
$$

in which $L(k)=2 \pi / k ; \bar{U}$ is provided by Eq. 21 .

(ii) If the current specified is the Stokes drift $U_{S}$, then Eq. 16 becomes the implicit algebraic equation

$$
f(k)=0=\frac{L(k)}{T}-\frac{Q(k)}{h}-U_{S}
$$

where again $L(k)=2 \pi / k ; Q$ is provided by Eq. 22 .

Field Kinematics. The final application step is the prediction of the complete kinematics. The water surface elevation is Eq. 19 and the velocity components are Eqs. 26 and 27. In the fixed frame, the horizontal velocity is $u=U+C$.

The acceleration components are

$$
\begin{aligned}
& \frac{D u}{D t}=U \frac{\partial U}{\partial X}+w \frac{\partial U}{\partial z} \\
& \frac{D w}{D t}=U \frac{\partial w}{\partial X}+w \frac{\partial w}{\partial z}=U \frac{\partial U}{\partial z}-w \frac{\partial U}{\partial X}
\end{aligned}
$$

where $\partial w / \partial X=\partial U / \partial z$ from the irrotational flow constraint, $\partial w / \partial z=-\partial U / \partial X$ from the 
local mass conservation equation, and

$$
\begin{aligned}
& \frac{\partial U}{\partial X}=(g k)^{1 / 2} \sum_{i=1}^{N} \varepsilon^{i} \sum_{j=1}^{i} j^{2} A_{i j} \frac{\cosh j k(h+z)}{\cosh j k h} \sin j k X \\
& \frac{\partial U}{\partial z}=(g k)^{1 / 2} \sum_{i=1}^{N} \varepsilon^{i} \sum_{j=1}^{i} j^{2} A_{i j} \frac{\sinh j k(h+z)}{\cosh j k h} \cos j k X
\end{aligned}
$$

The dynamic pressure is

$$
p_{d}(X, z)=\rho R-\frac{1}{2} \rho\left(U^{2}+w^{2}\right)
$$

Figure 2 provides an illustration of the predictive capability of the Stokes fifth order theory. The wave conditions are $h=100 \mathrm{~m}, T=10 \mathrm{~s}, H=10 \mathrm{~m}$ and $U_{E}=0$, as in Appendix B. The Stokes V predictions for water surface elevation, and horizontal velocity and horizontal acceleration along the water surface are compared with predictions from Fourier Approximation wave theory (Sobey, 1989) at truncation order 18. Agreement is visually perfect.

Limits of Validity. There is an expectation that the limit of validity may be restricted to waves of moderate height in deeper water. There is no strong expectation that the precision will extend to the immediate neighbourhood of the limit wave, or that the Stokes-style theory will extend very far into shallow water.

The limit wave is expected to have a slope $\partial \eta / \partial x$ discontinuity at the crest. Eq. 19 for the water surface is smooth and continuous, precluding a crest discontinuity. The dominant length scale transitions from $L$ to $h$ in transitional and shallow water, but it is also known (e.g. Williams, 1985) that very high order Stokes-style theories can push the transitional to shallow limits somewhat.

The limits of the present fifth-order theory are now investigated, over a range of dimensionless depths $\omega^{2} \mathrm{~h} / \mathrm{g}$ from 0.6 (shallow-transitional) to 4.0 (deep) and a range of dimensionless wave heights $\omega^{2} H / g$ from almost zero to the limit wave. For progressive waves, Williams (1985) provides tabulated predictions of the limit wave height $H_{\text {Limit }}$ for twenty-two $\omega^{2} h / g$ depths between 0.06 and 12.0. The theory is Stokes-style and computer extended to very high orders. A rational approximation to these tabulated predictions

$$
\left.\frac{\omega^{2} H_{\text {Limit }}}{g}\right|_{\text {Progressive }}=c_{0} \tanh \frac{a_{1} p+a_{2} p^{2}+a_{3} p^{3}}{1+b_{1} p+b_{2} p^{2}}
$$

where $p=\omega^{2} h / g, a_{1}=0.7879, a_{2}=2.0064, a_{3}=-0.0962, b_{1}=3.2924, b_{2}=-0.2645$ and $c_{0}=1.0575$, has a maximum error of 0.0014 over the range of the tables. Theoretically, $\omega^{2} \mathrm{H} / \mathrm{g}$ reaches an asymptotic maximum of 1.0575 in deep water, falling to about 0.4 at $\omega^{2} h / g=0.6$. A predicted limit wave height for each $\omega^{2} h / g$ is available from Eq. 41.

Predicted field variables often provide explicit measures of suspect validity, and routine perusal of field solution surfaces for $\eta, u, w, D u / D t, D w / D t$ and $p_{d}$ is always a good 

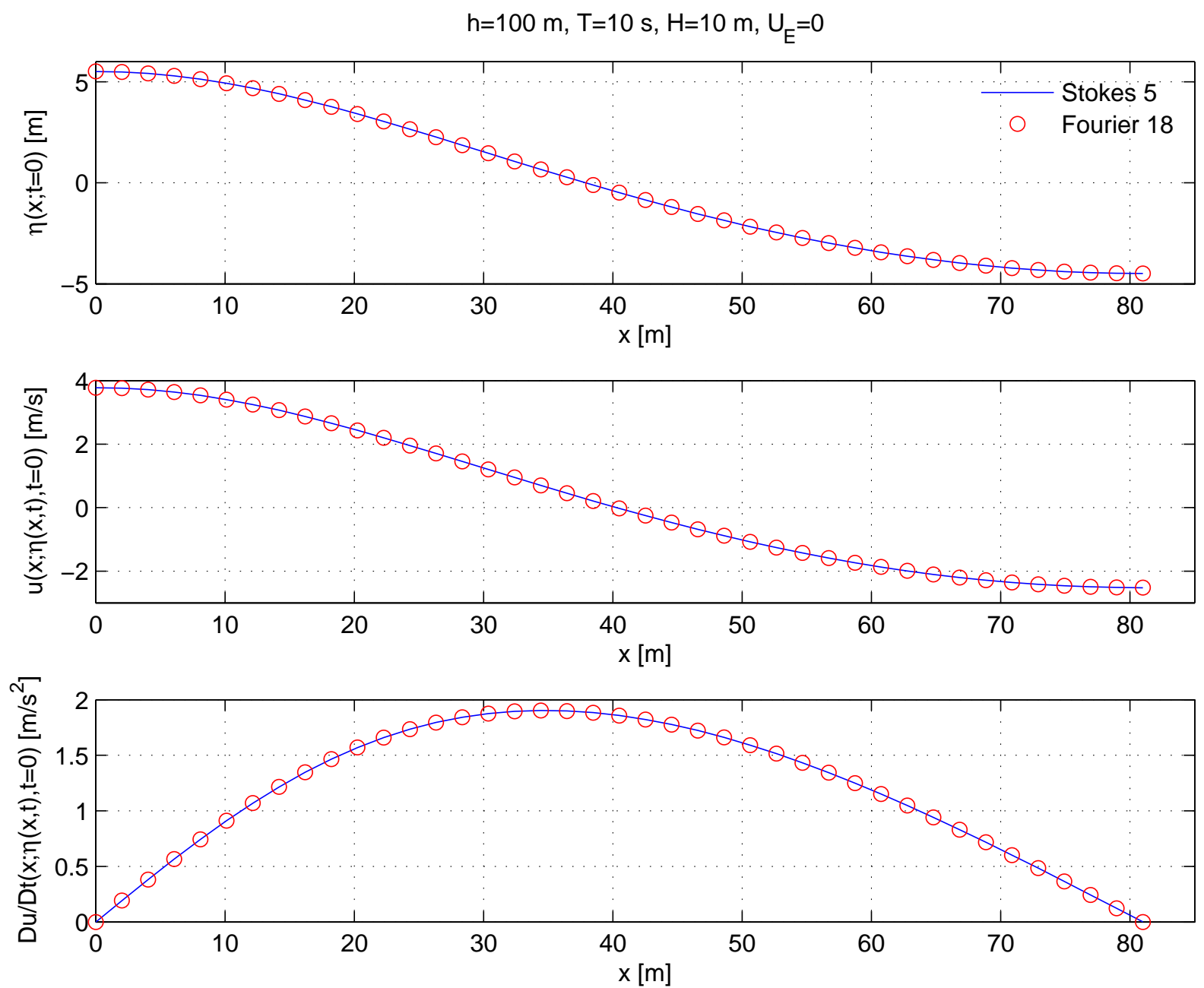

Figure 2: Stokes 5 and Fourier 18 predictions for water surface, and horizontal velocity and acceleration at water surface for $h=100 \mathrm{~m}, T=10 \mathrm{~s}, H=10 \mathrm{~m}$ and $U_{E}=0$.

practice. For progressive waves, early warning of suspect validity is often provided by a lack of smoothness in the water surface profile and/or kinematics along the water surface.

The more focused kinematic measures in Figures 3a through d provide a quite explicit view of the limits of validity. Figure 3 a shows the predicted evolution of the wave length $L / h$ or aspect ratio of the solution field. $L / h$ rapidly falls as the depth $\omega^{2} h / g$ increases. There is a small increase in $L / h$ with increasing wave height. Both trends are the expected response patterns, and there is no hint of suspect validity in this response.

Figure $3 \mathrm{~b}$ shows the predicted evolution of the root-mean-square error $\omega^{3} f_{K} / g^{2}$ (see Eq. 31a) in the kinematic free surface boundary condition. As expected, the rms error does increase with wave height. The variation with depth is smaller, but there is a suggestion that the rms error becomes somewhat larger as shallow water is approached. This is a gentle hint of increasingly unsatisfactory response at transitional to shallow depths. A 


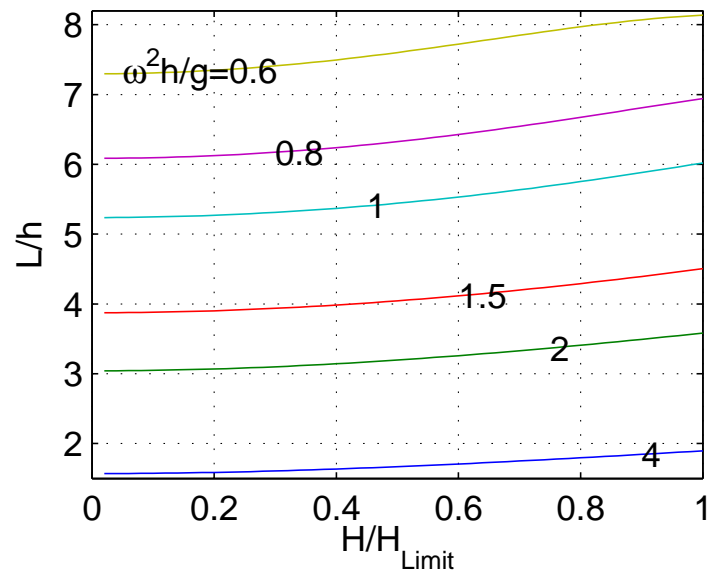

(a) Wave Length

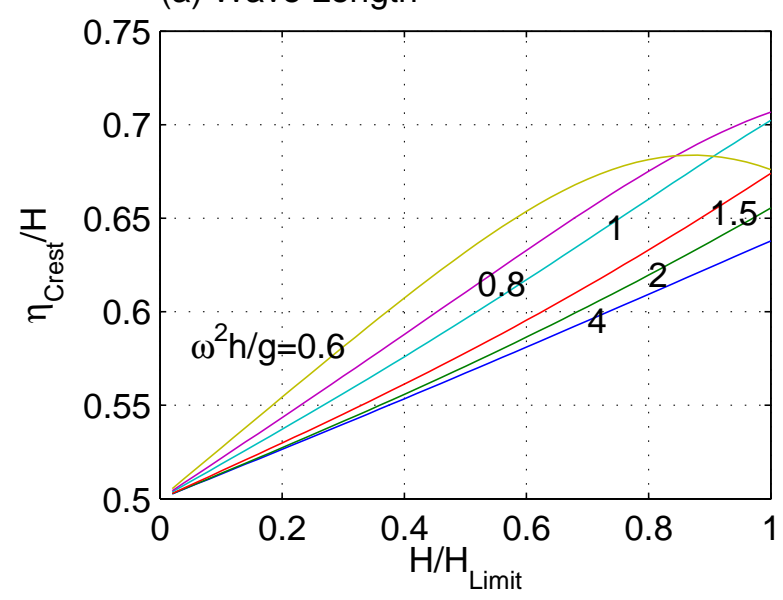

(c) Crest Elevaltion

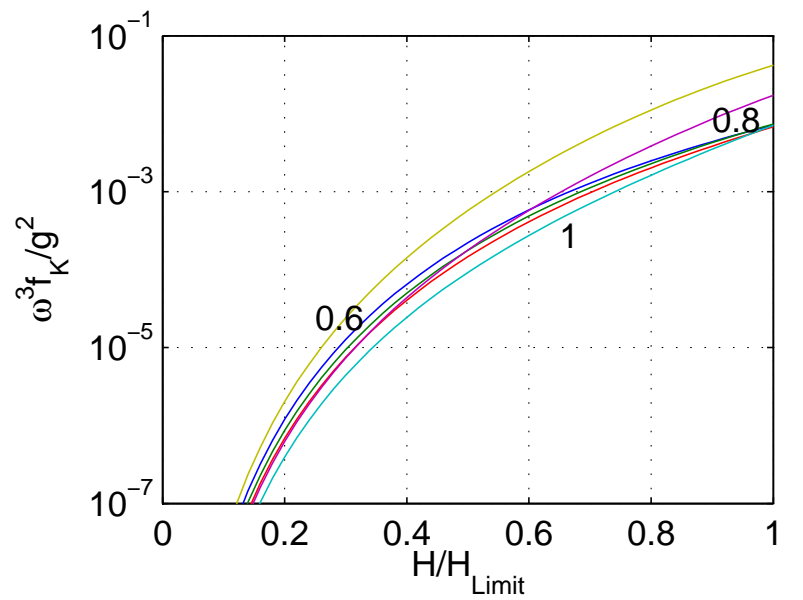

(b) rms Error in KFSBC

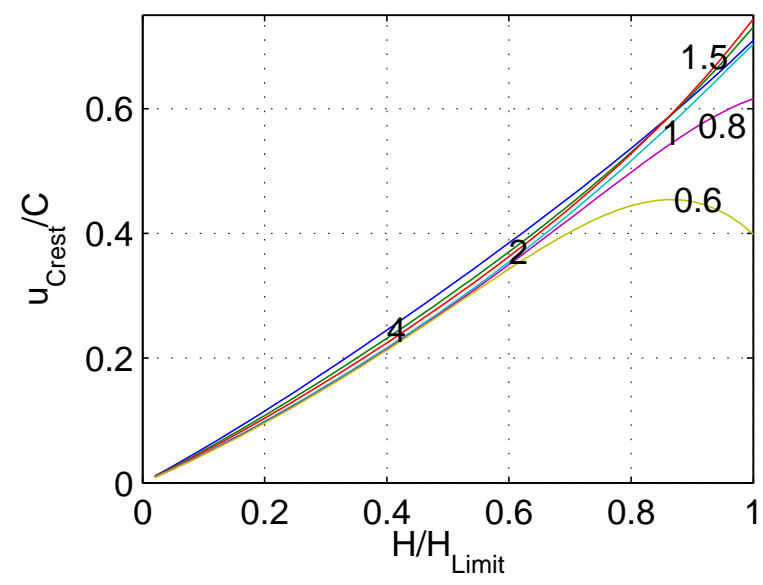

(d) Crest Velocitv

Figure 3: Stokes 5 predictions for evolution of selected integral and field variables.

very similar trend is exhibited by the dynamic free surface boundary condition.

Figure $3 c$ addresses the crest elevation $\eta_{\text {Crest }} / H$, where $\eta_{\text {Crest }}$ is $\eta(x=0, t=0)$. Experience with non-linear progressive waves suggests that the crest elevation climbs above $0.5 \mathrm{H}$, increasing with increasing wave height. That is indeed observed in Figure 3c for deep water wave heights to $\omega^{2} H / g \approx 1$. For wave heights beyond $\omega^{2} H / g \approx 1$, there is a trend reversal. As the limit wave is approached, there is the hint of a progression toward a maximum at $\omega^{2} h / g=0.8$, and a definite maximum well before the limit wave for $\omega^{2} h / g=0.6$.

Figure $3 \mathrm{~d}$ addresses the crest velocity $u_{\text {Crest }} / C$, where $u_{C r e s t}$ is $u(x=0, z=\eta, t=$ 0 ). Experience with non-linear progressive waves again suggests that the crest velocity increases toward the phase speed $C$ as the wave height increases. A common criterion for wave breaking has $u_{\text {Crest }} / C=1$. Figure $3 \mathrm{~d}$ closely repeats the trend pattern observed in Figure 3c, where there is a strong suggestion of valid predictions for $\omega^{2} h / g \gtrsim 1$, and an equally strong suggestion of suspect validity for $\omega^{2} h / g \lesssim 1$. 

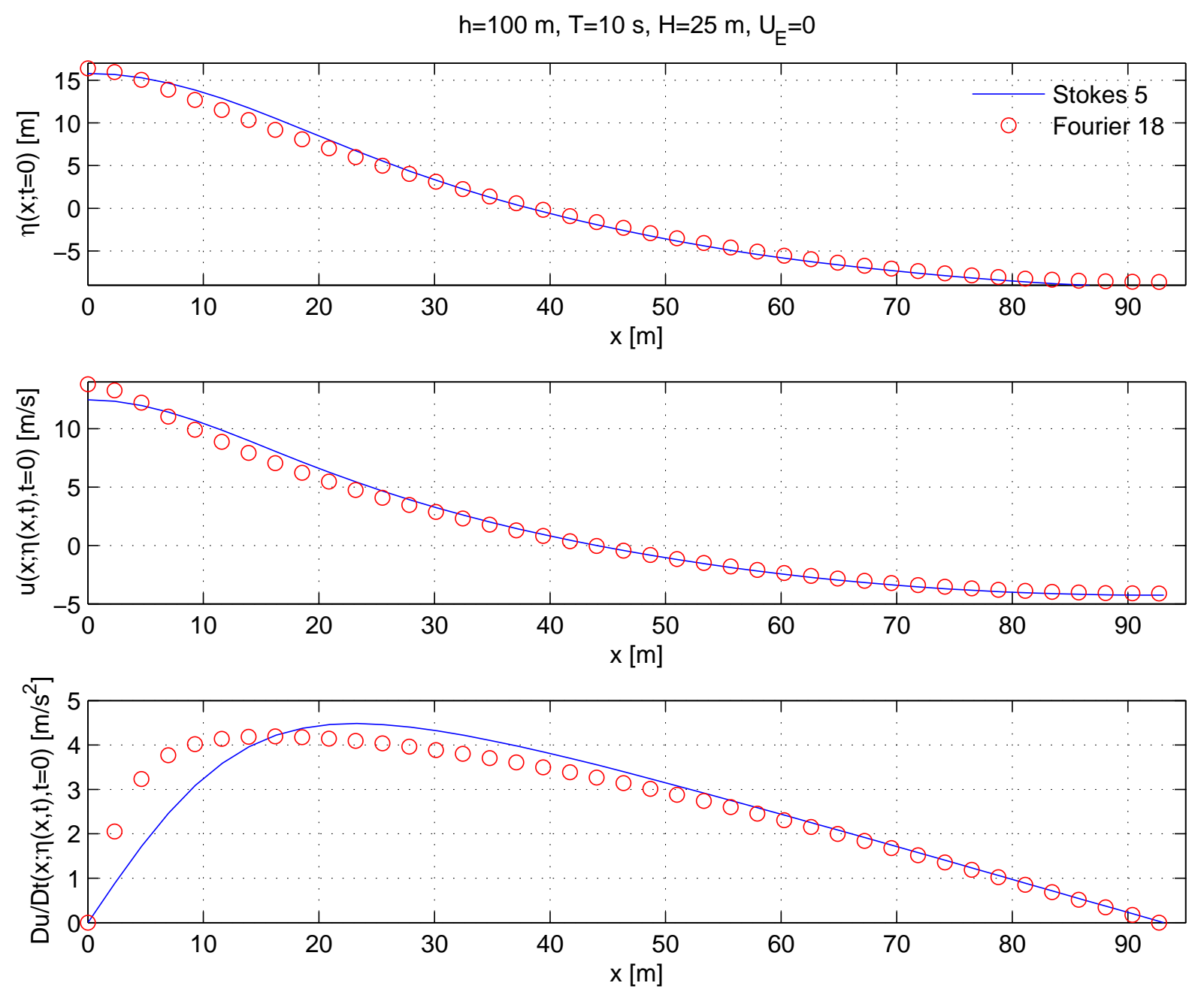

Figure 4: Stokes 5 and Fourier 18 predictions for water surface, and horizontal velocity and acceleration at water surface for $h=100 \mathrm{~m}, T=10 \mathrm{~s}, H=25 \mathrm{~m}$ and $U_{E}=0$.

What Figures 3a through d do not show is any hint of suspect validity as the limit wave is approached in deep water, $\omega^{2} h / g \gtrsim 2$. The Stokes 5 predictions remain smooth and visually credible, as observed in Figure 4 for $h=100 \mathrm{~m}, T=10 \mathrm{~s}, H=25 \mathrm{~m}$ and $U_{E}=0$. These conditions are similar to Figure 2 except for the wave height which is quite close to the Williams (1985)-predicted limit wave of $26.1 \mathrm{~m}$ for these conditions. It is only on comparison with an arguably more credible prediction (Fourier 18) in this same figure that the validity of the Stokes 5 prediction is questioned. 


\section{Cnoidal Waves to Fifth Order}

The rationale for the cnoidal approximation to nonlinear steady waves is provided by the observation (Eq. 2) that the water depth $h$ is the dominant length scale in shallow water. Wave length and wave height are influential through the dimensionless groupings $L / h$ and $H / h$ respectively, where $L / h$ is understood to be moderately large. With anticipated length scale $h$, the appropriate time scale is $(h / g)^{1 / 2}$,

Following Lamb (1932, p.423-4), a suitable shallow-water focussed solution to the field Laplace Eq. 3 is the series

$$
\Psi(X, z)=(h+z) \frac{d F(X)}{d X}-\frac{(h+z)^{3}}{3 !} \frac{d^{3} F(X)}{d X^{3}}+\frac{(h+z)^{5}}{5 !} \frac{d^{5} F(X)}{d X^{5}}-\ldots
$$

which exactly satisfies both the field equation (Eq. 3) and the bottom boundary condition (Eq. 5). The function $F(X)$, the water surface $\eta(X)$ and the balance of the solution remains to be determined from the balance of the boundary constraints.

Literature shallow water wave theories (Keller, 1948; Laitone, 1960; Fenton, 1979) also introduce a small and dimensionless straining parameter $\alpha$ such that all variation with $X$ and $z$ are as

$$
\chi=\alpha X / h, \quad \zeta=z / h
$$

respectively.

The shallow water series, Eq. 42, becomes

$$
\Psi(X, z)=\left(g h^{3}\right)^{1 / 2}\left[(1+\zeta) f(\chi)-\frac{(1+\zeta)^{3}}{3 !} \alpha^{2} \frac{d^{2} f}{d \chi^{2}}+\frac{(1+\zeta)^{5}}{5 !} \alpha^{4} \frac{d^{4} f}{d \chi^{4}}-\ldots\right]
$$

which continues to satisfy both the field Laplace equation and the bottom boundary condition.

Analytical expansions are adopted for the residual dependent variables, respectively

$$
f(\chi)=\sum_{i=0}^{N} \varepsilon^{i} \sum_{j=0}^{i} A_{i j} \mathrm{cn}^{2 j}(\chi ; m)
$$

for $f(\chi)$,

$$
\eta(X)=h \eta(\chi)=h \sum_{i=0}^{N} \varepsilon^{i} \sum_{j=0}^{i} B_{i j} \operatorname{cn}^{2 j}(\chi ; m)
$$

for the water surface elevation, and

$$
\begin{gathered}
R=g h\left[C_{0}+\sum_{i=1}^{N} C_{i} \varepsilon^{i}\right] \\
\bar{U}=(g h)^{1 / 2}\left[D_{0}+\sum_{i=1}^{N} D_{i} \varepsilon^{i}\right] \\
Q=\left(g h^{3}\right)^{1 / 2}\left[E_{0}+\sum_{i=1}^{N} E_{i} \varepsilon^{i}\right]
\end{gathered}
$$


for the Bernoulli constant, mean fluid speed and volume flux respectively. The $A, B$, $C, D$ and $E$ are dimensionless coefficients. Note the strong analogy with the deep water expansions in Eqs. 19 through 22.

Existing theories by Laitone (1960) to $N=2$ and Fenton (1979) to $N=9$ adopt the trough depth $d$ (see Figure 1) rather the MWL depth $h$ as the length scale. The Laitone theory is based on the Euler equations. Fenton adopts $\alpha^{2}$ as the expansion parameter. Neither contribution assumes a $\mathrm{cn}^{2 j}(\chi)$ dependence but establish ordinary differential equations for $f(\chi)$ at each order whose solution provides the $\mathrm{cn}^{2 j}(\chi)$ dependence. This $\mathrm{cn}^{2 j}(\chi)$ dependence is directly adopted in Eqs. 45 and 46.

The Jacobian elliptic cosine $\mathrm{cn}(\chi ; m)$ has a period of $4 \mathrm{~K}(m)$, where $\mathrm{K}(m)$ is the Jacobian elliptic integral of the first kind with parameter $m$. Accordingly, $\mathrm{cn}^{2}(\chi ; m)$ has period $2 \mathrm{~K}(m)$. Imposing the periodic lateral boundary condition, Eq. 8, on the $f(\chi)$ and $\eta(\chi)$ expansions defines the straining parameter as

$$
\alpha=\frac{2 \mathrm{~K}(m) h}{L}
$$

$\alpha$ or $L$ remains an unknown, for which an analytical expansion must be adopted. As only $\alpha^{2}$ appears directly in the analytical solution, in Eq. 45, an expansion for $\alpha^{2}$ is adopted:

$$
\alpha^{2}=\left[F_{0}+\sum_{i=1}^{N} F_{i} \varepsilon^{i}\right]
$$

in which $F_{i}$, like $C_{i}$ through $E_{i}$, is a dimensionless coefficient.

Of the dimensionless groupings identified in Eq. 2, the primary grouping $h / L$ is implicit in the $\mathrm{cn}^{2 j}(\chi)$ dependence in Eqs. 45 and 46, and also in Eq. 51. It is convenient to adopt the secondary parameter $H / h$ in the definition of the expansion parameter:

$$
\varepsilon=\frac{H}{h}
$$

At zeroth order $\left(\varepsilon^{0}\right)$ again, there is no wave, $\eta(X) \equiv 0$, the steady flow is uniform and critical, and Eq. 24 is applicable. For shallow water waves at very small wave height, the phase speed $C$ is $(g h)^{1 / 2}$. Accordingly,

$$
C_{0}=\frac{1}{2}, \quad D_{0}=1, \quad E_{0}=1, \quad F_{0}=0
$$

By definition, the Jacobian elliptic $\mathrm{cn}^{2}(\chi ; m)$ function is always positive. The mean water level constraint (Eq. 10) is not automatically satisfied as in the Stokes theory, but must be imposed as an independent equation. Eq. 10 becomes

$$
\int_{0}^{\mathrm{K}(m)} \eta(\chi ; m) d \chi=0=\sum_{i=0}^{N} \varepsilon^{i} \sum_{j=0}^{i} B_{i j} \int_{0}^{\mathrm{K}(m)} \mathrm{cn}^{2 j}(\chi ; m) d \chi
$$


Analytical integration of the definite integrals follows from the recurrence relationship

$$
\begin{gathered}
\quad(2 j-1) m \mathbf{I}_{j}=-(2 j-2)(1-2 m) \mathbf{I}_{j-1}+(2 j-3)(1-m) \mathbf{I}_{j-2} \\
\text { where } \mathbf{I}_{j}=\int_{0}^{\mathrm{K}(m)} \operatorname{cn}^{2 j}(\chi ; m) d \chi, \quad \mathbf{I}_{0}=\mathrm{K}(m), \mathbf{I}_{1}=\frac{e+m-1}{m} \mathrm{~K}(m)
\end{gathered}
$$

established from Gradshteyn \& Ryzhik (1965, §5.131(2)). Also

$$
e=\frac{\mathrm{E}(m)}{\mathrm{K}(m)}
$$

in which $\mathrm{E}(m)$ is the Jacobian elliptic integral of the second kind with parameter $m$.

In addition, the underlying steady flow part of the stream function, $-\bar{U}(h+z)$ as in Eq. 18 for the Stokes theory, is part of the initial term of the shallow water series, Eq. 44, for $\Psi$ in the cnoidal theory. It must be separately identified because of its fundamental role in the dispersion relationship Eq. 15. From Eqs. 11a

$$
\begin{aligned}
\bar{U} & =-\frac{2}{L} \int_{0}^{L / 2} \frac{\partial \Psi}{\partial z} d X=-(g h)^{1 / 2} \frac{1}{\mathrm{~K}(m)} \int_{0}^{\mathrm{K}(m)} f(\chi) d \chi \\
& =-(g h)^{1 / 2} \sum_{i=0}^{N} \varepsilon^{i} \sum_{j=0}^{i} A_{i j} \frac{1}{\mathrm{~K}(m)} \int_{0}^{\mathrm{K}(m)} \operatorname{cn}^{2 j}(\chi ; m) d \chi
\end{aligned}
$$

Analytical integration of the definite integrals again follows Eq. 55.

In an analogous manner to the Stokes theory, Equations 44 through 57 are used in

(i) the wave height constraint (Eq. 9),

(ii) the mean water level constraint (Eq. 54),

(iii) identification of the mean fluid speed (Eq. 57),

(iv) the kinematic free surface boundary condition (Eq. 6), and

(v) the dynamic free surface boundary condition (Eq. 7).

Collecting the coefficients of powers of $\varepsilon$ defines the analytical problem at consecutive orders $1 \ldots N$, which provide sufficient independent algebraic equations to determine the balance of the $A_{i j}$ through $F_{i}$ coefficients.

At each order there are five algebraic coefficient equations, corresponding to constraints (i) through (v). The free surface boundary condition constraints, (iv) and (v), naturally contain powers and products of Jacobian $\operatorname{sn}(\chi), \operatorname{cn}(\chi)$ and $\operatorname{dn}(\chi)$ functions at each order, specifically

(a) at first order: $\operatorname{sn}^{2}(\chi), \operatorname{dn}^{2}(\chi)$

(b) at second order: $\operatorname{sn}^{4}(\chi), \operatorname{dn}^{4}(\chi) ; \operatorname{sn}^{2}(\chi) \operatorname{cn}^{2}(\chi), \operatorname{dn}^{2}(\chi) \operatorname{cn}^{2}(\chi), \operatorname{sn}^{2}(\chi) \operatorname{dn}^{2}(\chi)$ 
(c) at third order: $\operatorname{sn}^{6}(\chi), \mathrm{dn}^{6}(\chi) ; \operatorname{sn}^{2}(\chi) \mathrm{cn}^{4}(\chi), \mathrm{dn}^{2}(\chi) \mathrm{cn}^{4}(\chi), \operatorname{sn}^{2}(\chi) \mathrm{dn}^{4}(\chi)$; $\operatorname{sn}^{2}(\chi) \operatorname{cn}^{2}(\chi) \operatorname{dn}^{2}(\chi)$

(d) and similar for higher orders.

These power and product terms are expanded as sums of powers of $\mathrm{cn}^{2}(\chi)$, using identities among these functions based on $\operatorname{sn}^{2}(\chi)=1-\mathrm{cn}^{2}(\chi)$ and $\operatorname{dn}^{2}(\chi)=1-m+m \mathrm{cn}^{2}(\chi)$ [Gradshteyn \& Ryzhik $(1965, \S 8.154(4,5))]$. The listed boundary constraints become double power series in $\varepsilon$ and $\mathrm{cn}^{2}(\chi ; m)$. All terms have the form $\varepsilon^{i} \mathrm{cn}^{2 j}(\chi ; m)$ where $i, j=0,1,2,3, \ldots$.

As the boundary constraints must be satisfied for all $X$, the coefficients terms must also be zero. At each order, the number of unknown coefficients and the number of coefficient equations are balanced. But starting at second order, the highest order coefficient equations for $\mathrm{cn}^{2 j}(\chi)$ from the free surface boundary constraints (iv) and (v) are not independent. The coefficient $B_{22}$ is provided from the third order coefficient equations. In turn, $B_{32}$ and $B_{33}$ is completed at fourth order, $B_{42}$ to $B_{44}$ at fifth order, and $B_{52}$ to $B_{55}$ at sixth order. Given the Eq. 53 solution for the zeroth order, all subsequent coefficient equations are linear and algebraic, leading to a unique solution and the definition of the dimensionless coefficients $A_{i j}, B_{i j}, C_{i}, D_{i}, E_{i}$ and $F_{i}$. Routine use was made of computer algebra software. The resulting coefficients to fifth order are listed in Appendix C.

Field Kinematics. The code prediction of the field kinematics is a rather more challenging problem than for deep water waves. While the shallow water series is accessible analytically and on a computer algebra platform, this simplicity is partially lost on a computational platform where analytical differentiation is not available. A preparatory step is to re-write Eq. 44 as

$$
\begin{gathered}
\Psi(\chi, \zeta)=\sum_{k=0}^{4}(-1)^{k} \frac{(1+\zeta)^{2 k+1}}{(2 k+1) !} f_{k}(\chi) \\
\text { in which } f_{0}(\chi)=f(\chi) \text { and } f_{k}(\chi)=\alpha^{2} \frac{d^{2}}{d \chi^{2}} f_{k-1}, k=1, \ldots 4
\end{gathered}
$$

The summation truncation at 4 includes all terms to fifth order. Each of the $f_{k}(\chi)$ is formally similar to Eq. 45, being

$$
\begin{gathered}
f_{k}(\chi)=\sum_{i=0}^{N} \varepsilon^{i} \sum_{j=0}^{i} A_{i j}^{k} \mathrm{cn}^{2 j}(\chi ; m) \\
\text { in which }\left[A_{i j}^{0}\right]=\left[A_{i j}\right] \text { and }\left[A_{i j}^{k}\right]=\left[F_{i}\right]\left[A_{i j}^{k-1}\right] \mathbb{F} \text { for } k=1 \ldots 4
\end{gathered}
$$


$\left[A_{i j}^{k}\right]$ is the $6 \times 6$ matrix with row $i$ and column $j$ coefficients $A_{i j}^{k}$. $\left[A_{i j}\right]$ is the matrix of coefficients defined in Eq. 45, namely

$$
\left[A_{i j}\right]=\left[\begin{array}{cccccc}
A_{00} & 0 & 0 & 0 & 0 & 0 \\
A_{10} & A_{11} & 0 & 0 & 0 & 0 \\
A_{20} & A_{21} & A_{22} & 0 & 0 & 0 \\
A_{30} & A_{31} & A_{32} & A_{33} & 0 & 0 \\
A_{40} & A_{41} & A_{42} & A_{43} & A_{44} & 0 \\
A_{50} & A_{51} & A_{52} & A_{53} & A_{54} & A_{55}
\end{array}\right]
$$

$\mathbb{F}$ is the $6 \times 6$ transformation matrix that provides the $d^{2} / d \chi^{2}$ operation. $\left[F_{i}\right]$ is the transformation matrix that provides multiplication by the $\alpha^{2}$ expansion. Both are listed in Appendix E.

The product $\left[A_{i j}^{k-1}\right] \mathbb{F}$ is a classical matrix multiplication of square $6 \times 6$ matrices. Each column of $\left[A_{i j}^{k-1}\right]$ or $\mathbb{F}$ or the product $\left[A_{i j}^{k-1}\right] \mathbb{F}$ is also a vector of coefficients of $\operatorname{cn}^{2 j}(\chi)$, so that multiplication by $\left[F_{i}\right]$ involves six separate polynomial multiplications, truncated to the six leading terms.

Prediction of $w$ and $\partial U / \partial X$ also requires an additional sequence of functions $g_{k}(\chi)$, where

$$
\begin{aligned}
g_{0}(\chi) & =\frac{d}{d \chi} f_{0}(\chi)=\sum_{i=0}^{N} \varepsilon^{i} \sum_{j=0}^{i} A_{i j}^{0} \frac{d}{d \chi} \operatorname{cn}^{2 j}(\chi ; m) \\
& =\operatorname{scd}(\chi ; m) \sum_{i=0}^{N} \varepsilon^{i} \sum_{j=0}^{i} \mathbb{A}_{i j}^{0} \operatorname{cn}^{2 j}(\chi ; m) \\
g_{k}(\chi) & =\frac{d}{d \chi} g_{k-1}(\chi)=\sum_{i=0}^{N} \varepsilon^{i} \sum_{j=0}^{i} \mathbb{A}_{i j}^{k} \frac{d}{d \chi} \operatorname{scd}(\chi ; m) \operatorname{cn}^{2 j}(\chi ; m) \text { for } k=1 \ldots 4 \\
& =\operatorname{scd}(\chi ; m) \sum_{i=0}^{N} \varepsilon^{i} \sum_{j=0}^{i} \mathbb{A}_{i j}^{k} \operatorname{cn}^{2 j}(\chi ; m)
\end{aligned}
$$

in which $\left[\mathbb{A}_{i j}^{0}\right]=\left[A_{i j}\right] \mathbb{G}_{0}$ and $\left[\mathbb{A}_{i j}^{k}\right]=\left[F_{i}\right]\left[\mathbb{A}_{i j}^{k-1}\right] \mathbb{G}$ for $k=1 \ldots 4$

The function $\operatorname{scd}(\chi)$ is

$$
\operatorname{scd}(\chi)=\operatorname{sn}(\chi) \operatorname{cn}(\chi) \operatorname{dn}(\chi)
$$

The Jacobian elliptic functions $\operatorname{sn}(\chi)$ and $\operatorname{dn}(\chi)$ appear only in this product combination in the cnoidal theory. The transformation matrices $\mathbb{G}_{0}$ and $\mathbb{G}$ are listed in Appendix E.

Accordingly, the velocity components $U, w$ are

$$
\begin{aligned}
& U(X, z)=\frac{\partial \Psi(X, z)}{\partial z}=(g h)^{1 / 2} \sum_{k=0}^{4}(-1)^{k} \frac{(1+\zeta)^{2 k}}{(2 k) !} f_{k}(\chi) \\
& w(X, z)=-\frac{\partial \Psi(X, z)}{\partial X}=-\alpha(g h)^{1 / 2} \sum_{k=0}^{4}(-1)^{k} \frac{(1+\zeta)^{2 k+1}}{(2 k+1) !} g_{k}(\chi)
\end{aligned}
$$


The acceleration components $D u / D t, D w / D t$ are available from Eq. 36 and 37, in which $\partial U / \partial X$ and $\partial U / \partial z$ are

$$
\begin{aligned}
& \frac{\partial U}{\partial X}=\alpha(g / h)^{1 / 2} \sum_{k=0}^{4}(-1)^{k} \frac{(1+\zeta)^{2 k}}{(2 k) !} g_{k}(\chi) \\
& \frac{\partial U}{\partial z}=(g / h)^{1 / 2} \sum_{k=1}^{4}(-1)^{k} \frac{(1+\zeta)^{2 k-1}}{(2 k-1) !} f_{k}(\chi)
\end{aligned}
$$

In the fixed frame, the horizontal velocity is $u=U+C$.

The dynamic pressure is available from Eq.40.

Confirmation of Theory. Confirmation of a verifiably correct analytical theory again follows from Richardson extrapolation to the limit. The details have been outlined in Eqs. 30 through 33. It is appropriate to observe that this procedure is dependent not just on the analytical theory but also its code implementation. As outlined in Eqs. 58 through 61 together with Appendix E, the necessary code implementation in the cnoidal theory is manifestly not trivial.

For $h=2 \mathrm{~m}, T=10 \mathrm{~s}, U_{E}=0, H_{1}=0.25 \mathrm{~m}$ and $H_{2}=0.5 \mathrm{~m}, \mu_{K}$ and $\mu_{D}$ are 6.257 and 6.346 respectively, confirming that the theory is correct to fifth order.

As an aide to confirmation of implementation code, a complete list of solution coefficients are given in Appendix D for a specific listed wave condition.

Dispersion. For the cnoidal theory, the dispersion relationship, Eqs. 15 or 16, must be solved for the Jacobian elliptic parameter $m$. In a common application, $h, H$ and $T$ are known.

(i) If the current specified is the Eulerian current $U_{E}$, then Eq. 15 becomes the implicit algebraic equation

$$
f(m)=0=\frac{L(m)}{T}-\bar{U}(m)-U_{E}
$$

in which $L(m)=2 \mathrm{~K}(m) h / \alpha(m)$ from Eq. $50 ; \alpha$ is provided by Eq. 51 and $\bar{U}$ by Eq. 48.

(ii) If the current specified is the Stokes drift $U_{S}$, then Eq. 16 becomes the implicit algebraic equation

$$
f(m)=0=\frac{L(m)}{T}-\frac{Q(m)}{h}-U_{S}
$$

where again $L(m)=2 \mathrm{~K}(m) h / \alpha(m) ; \alpha$ is provided by Eq. 51 and $Q$ by Eq. 49 .

For even moderately steep cnoidal waves, the Jacobian $m$ may be very close to one. It is numerically appropriate to rephrase Eqs. 67 and 68 in terms of $m_{1}=1-m$. 
$\mathrm{h}=2 \mathrm{~m}, \mathrm{~T}=10 \mathrm{~s}, \mathrm{H}=0.5 \mathrm{~m}, \mathrm{U}_{\mathrm{E}}=0$
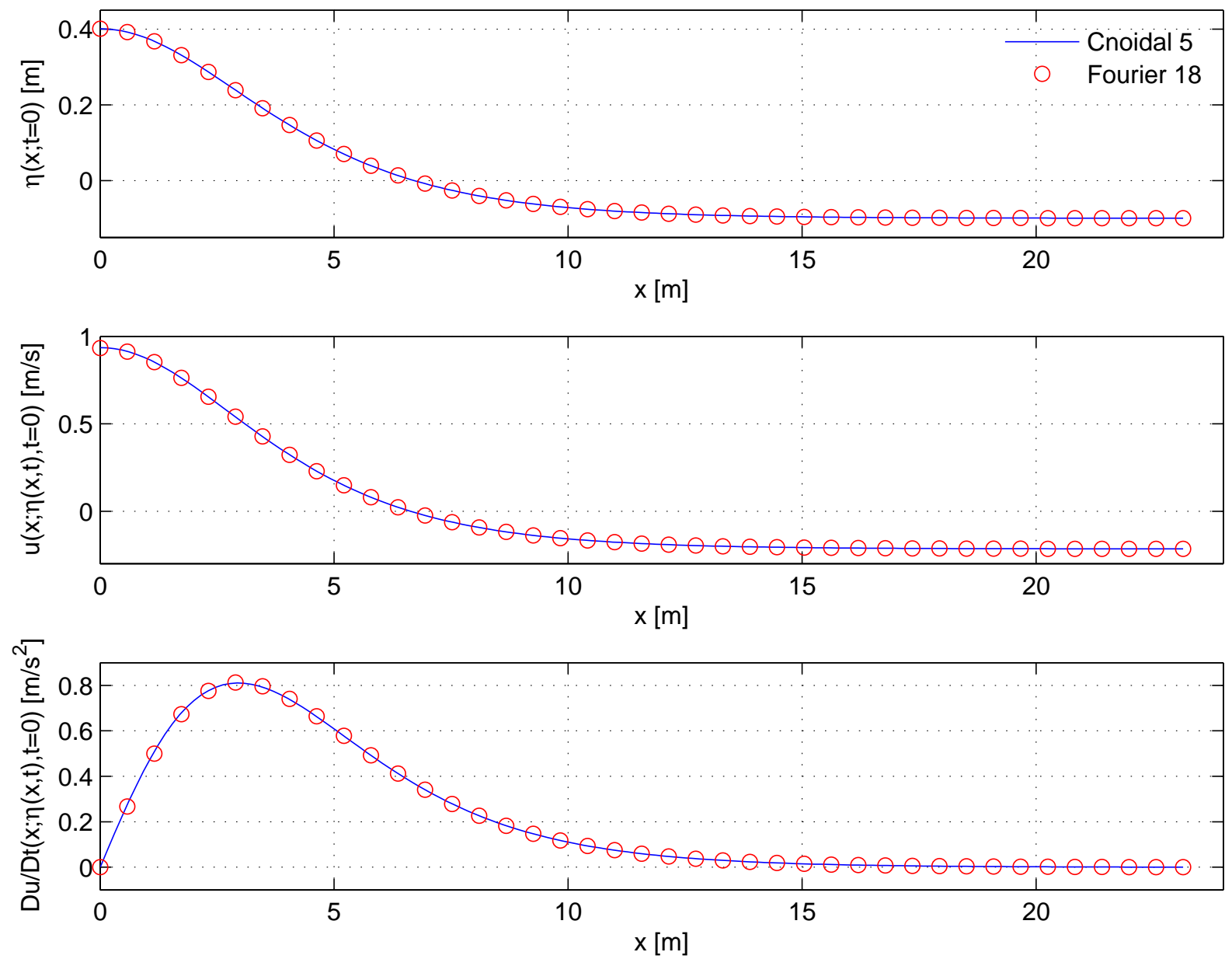

Figure 5: Cnoidal 5 and Fourier 18 predictions for water surface, and horizontal velocity and acceleration at water surface for $h=2 \mathrm{~m}, T=10 \mathrm{~s}, H=0.5 \mathrm{~m}$ and $U_{E}=0$.

Predicted Kinematics. Figure 5 provides an illustration of the predictive capability of the cnoidal fifth order theory. The wave conditions are $h=2 \mathrm{~m}, T=10 \mathrm{~s}, H=0.5 \mathrm{~m}$ and $U_{E}=0$, as in Appendix D. The cnoidal predictions for water surface elevation, and horizontal velocity and horizontal acceleration along the water surface are compared with the predictions from Fourier Approximation wave theory (Sobey, 1989) at truncation order 18. Agreement is visually perfect.

Limits of Validity. There is no expectation that the limits of validity of the cnoidal shallow water theory will extend too far into transitional depths or to wave heights approaching the limit wave.

The limits of the present fifth-order cnoidal theory are now investigated, over a range of dimensionless depths $\omega^{2} h / g$ from 0.05 (very shallow) to beyond 1.0 (transitional) and 


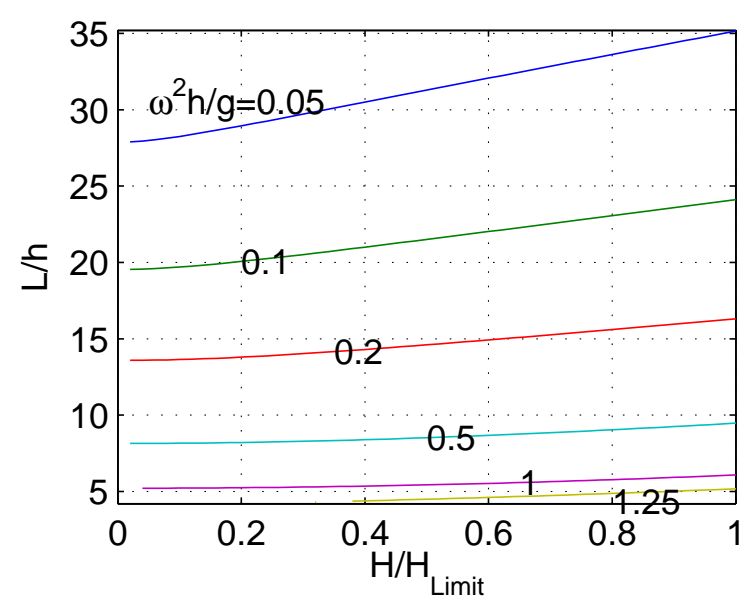

(a) Wave Length

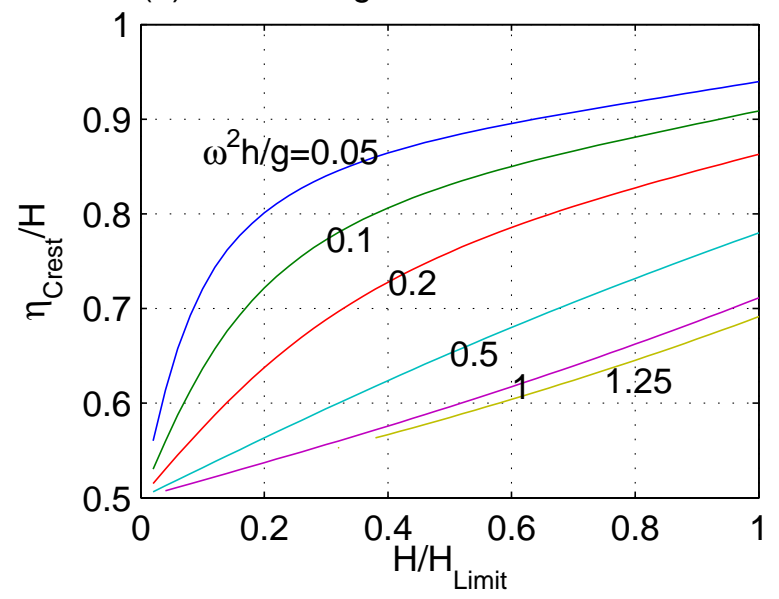

(c) Crest Elevaltion

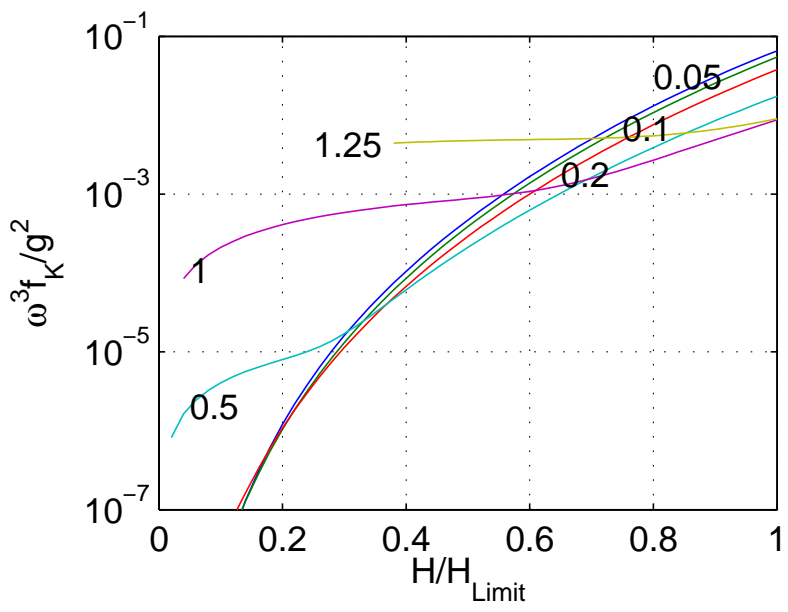

(b) rms Error in KFSBC

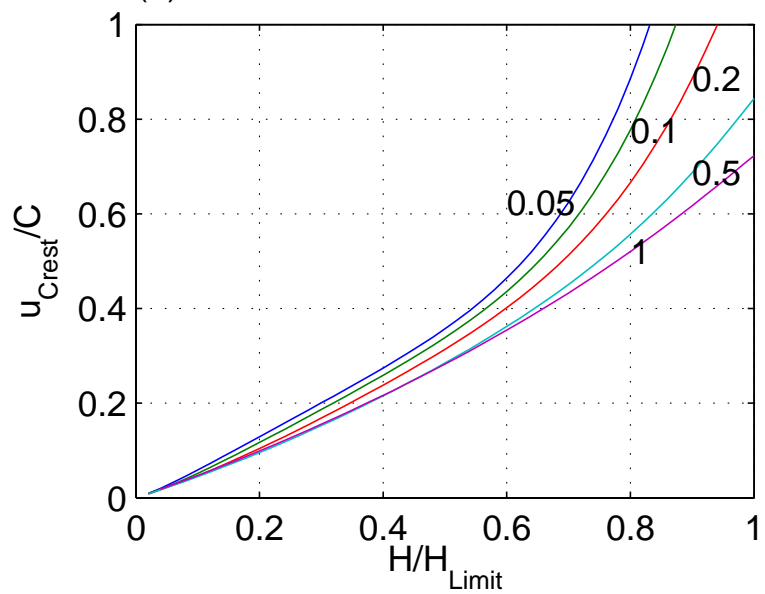

(d) Crest Velocitv

Figure 6: Cnoidal 5 predictions for evolution of selected integral and field variables.

a range of dimensionless wave heights $\omega^{2} H / g$ from almost zero to the Williams (1985)predicted limit wave, Eq. 41.

Attention is directed initially to the integral and field variables identified in Figure 3 for the Stokes theory, namely the wave length $L / h$, the rms error $\omega^{3} f_{K} / g^{2}$ in the kinematic free surface boundary condition, the crest elevation $\eta_{C r e s t} / H$ and the crest velocity $u_{C r e s t} / C$, in Figures 6a through d respectively. Figure 6a for the wave length could have been anticipated from Figure 3a. The wave length $L / h$ or aspect ratio of the solution field continues to increase as the depth decreases. Such long waves were indeed the basis for the shallow water approximation to the stream function, Eq. 42. The smaller increase in wave length with increasing wave height is again observed, becoming somewhat more intensified as the water shallows. The predictions for $\omega^{2} h / g=1.25$ are incomplete. For smaller wave heights, there was no solution to the dispersion relationship, Eq. 67. For $\omega^{2} h / g \gtrsim 1.5$, there was no solution for any wave height. This was expected from the shallow water focus 
of the theory imposed through adoption of the Eq. 42 solution for the field equation.

For depths $\omega^{2} h / g \lesssim 0.2$ clearly within the expected validity range for the cnoidal theory, the rms error responds (Figure 6b) almost exactly as for the Stokes theory (Figure 3b). Some hint of an approach to the no solution status at $\omega^{2} h / g=1.25$ in Figure 6a is apparent in the smaller wave height response at $\omega^{2} h / g=0.5,1$ and finally 1.25.

Figure $6 \mathrm{c}$ for the crest elevation provides no hint on any suspect validity of the cnoidal theory until well into transitional depths.

Figure $6 \mathrm{~d}$ for the crest velocity is seemingly well behaved but nevertheless predicts crest velocities well beyond the phase speed $C$ for shallow water waves less that the Williams (1985)-predicted limit wave height. Although it is not shown, the maximum acceleration along the water surface likewise increases smoothly well beyond the gravitational acceleration $g$ for waves less than the limit wave. While water surface profile predictions remain smooth and credible, as partially anticipated by Figure 6c, velocities, accelerations and dynamic pressure along the water surface do very clearly flag suspect validity with increasing wave height.

Figure 7 provides an illustration of declining validity for waves closer to the limit wave. The wave conditions are $h=2 \mathrm{~m}, T=10 \mathrm{~s}, H=1.0 \mathrm{~m}$ and $U_{E}=0$, differing from Figure 5 only in the wave height. The Williams (1985)-predicted limit wave is $1.59 \mathrm{~m}$ for these conditions. The cnoidal predictions for water surface elevation, and horizontal velocity and horizontal acceleration along the water surface are again compared with the predictions from Fourier Approximation wave theory at truncation order 18. The water surface predictions are visually perfect, but there are clear indications of flawed predictions with the horizontal velocity and acceleration at the water surface. This confirms the trends suggested by Figure $6 \mathrm{~b}$.

\section{Regions of Applicability}

It is apparent from the above discussions that the cnoidal theory is generally applicable in shallow water and the Stokes theory in deep water. There is an expectation of a region of overlapping applicability at transitional depths. It is apparent also that neither of the analytical theories remains valid on close approach to the limit wave.

Possible wave conditions extend over depths $h$ from almost zero to very large and over wave heights $H$ from very small to the limit wave $H_{\text {Limit }}$. Non-dimensionally, the region of application is $\Delta_{1}<\omega^{2} h / g \lesssim 4$ and $\Delta_{2}<H / H_{\text {Limit }}<1 ; \Delta_{1}$ is a small depth and $\Delta_{2}$ is a small wave height, both approaching zero in magnitude. Solutions were sought from both analytical theories for waves of period $10 \mathrm{~s}$, zero current, and depths of $0.5,1,2,3,4,5$, $10,15,20,30,40,50$ and $100 \mathrm{~m}$. Wave height at each depth ranged from almost zero to the Williams (1985)-predicted limit wave. For each analytical solution, predictions for

(i) water surface profile $\eta$

(ii) profiles along the water surface for $u, w, D u / D t$ and $p_{d}$ 

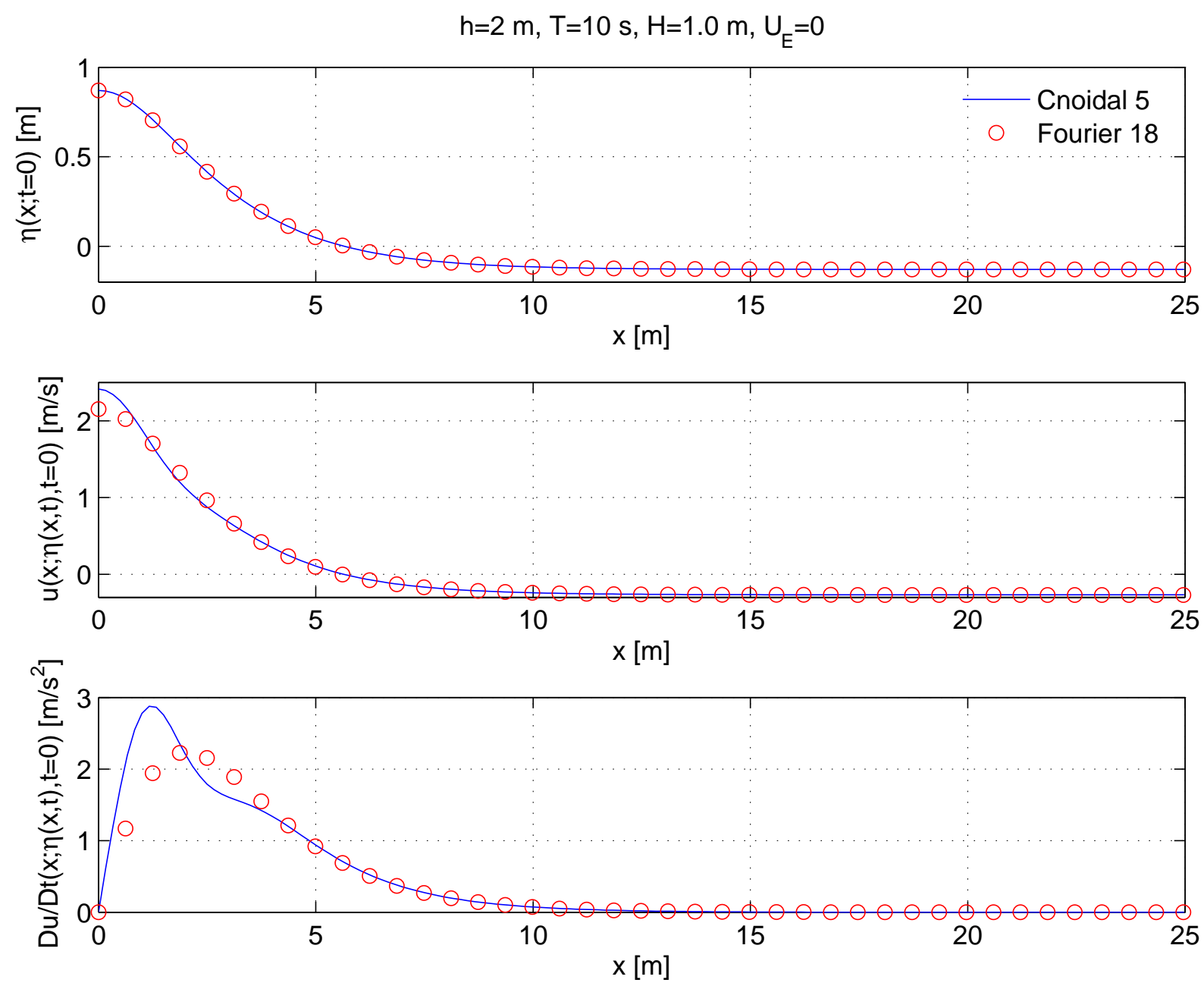

Figure 7: Cnoidal 5 and Fourier 18 predictions for water surface, and horizontal velocity and acceleration at water surface for $h=2 \mathrm{~m}, T=10 \mathrm{~s}, H=1.0 \mathrm{~m}$ and $U_{E}=0$.

(iii) complete field solutions for $u, w, D u / D t$ and $p_{d}$

were carefully perused. At each depth and for each theory, the highest visually-credible wave height was recorded. These are presented in Figure 8. In large measure, the trends apparent here reflected those anticipated from Figures 3 and 6 . The cnoidal theory extends from shallow to moderately-deep transitional depths. It does extend to moderately large wave heights but not to extreme wave height close to the limit wave. The Stokes theory extend from deep water to moderately-shallow transitional depths. It appears to extend to the limit wave in deep water, but this has been questioned in Figure 4. The applicability in transitional water is much more limited.

As expected, there is a region of overlapping application at transitional depths. The trend curves intersect at $\omega^{2} h / g \approx 0.65$, which suggests the adoption of cnoidal theory for $\omega^{2} h / g \lesssim 0.65$, and Stokes theory for $\omega^{2} h / g \gtrsim 0.65$. Fenton (1979) has suggested that 


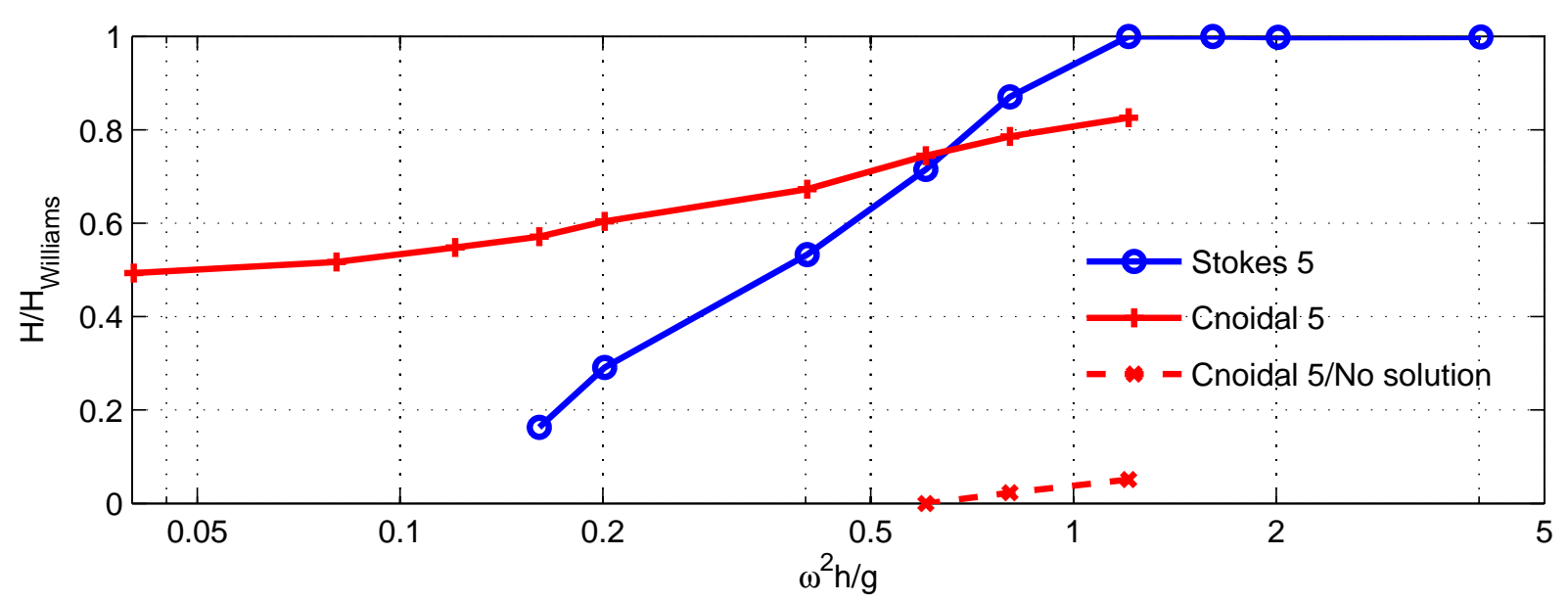

Figure 8: Comparative validity of Stokes 5 and cnoidal 5 analytical theories.

$L / h>8$ identifies the region of applicability for cnoidal wave theory. It is noted from Figures $3 \mathrm{a}$ and $6 \mathrm{a}$ that $L / h \approx 8$ is consistent with the present $\omega^{2} h / g \approx 0.65$ recommendation.

The dimensionless grouping called the Ursell Number

$$
\mathbf{U} \mathbf{r}=\frac{H / h}{(h / L)^{2}}=\frac{H L^{2}}{h^{3}}
$$

has found application in characterising nonlinear waves. Isobe et al. (1982) identify $\mathbf{U r} \approx$ 25 as typifying conditions where all three length scales are simultaneously influential. A Stokes analytical theory is suggested below this limit, and a cnoidal theory above this limit. This has provided useful guidance, though there is a suggestion from Figures 3a and 6a that perhaps wave height $H$ is not quite so prominent. There is the additional difficulty that $L$ is not known in advance, being part of the solution.

Figure 9 is a final illustration of this region of overlapping validity. In this application of both analytical theories, together with Fourier approximation wave theory at truncation order 18, the wave conditions are $h=15 \mathrm{~m}, T=10 \mathrm{~s}, H=6.0 \mathrm{~m}$ and zero current. The predictions for water surface elevation and for horizontal velocity along the water surface are quite close, but there is some clear separation with the horizontal acceleration predictions. This presumably follows from the manner in which higher order terms in the predictive series are accentuated for acceleration, and in a different manner for each of the predictions.

\section{Conclusions}

Analytical theories for steady progressive waves are based on a recognition of the dominant length scale appropriate for the flow. In deep water, the dominant length scale is the wave length $L$. A Stokes-style theory is a natural consequence. In shallow water, the dominant length scale is the water depth $h$. A cnoidal-style theory is a natural consequence. 

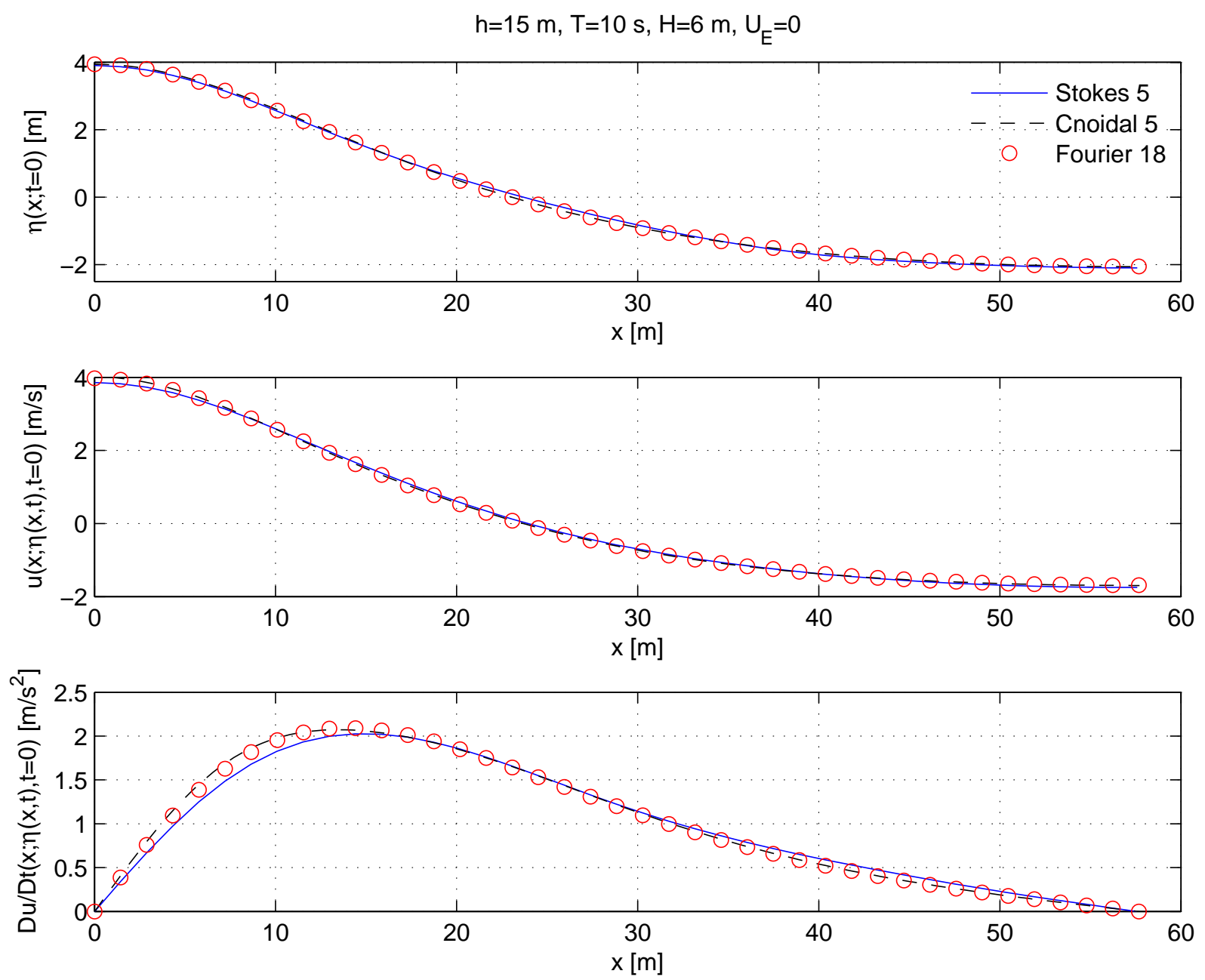

Figure 9: Stokes 5, cnoidal 5 and Fourier 18 predictions for water surface, and horizontal velocity and acceleration at water surface for $h=15 \mathrm{~m}, T=10 \mathrm{~s}, H=6.0 \mathrm{~m}$ and $U_{E}=0$.

It is emphasised nevertheless that these parallel Stokes and cnoidal analytical theories have most of their mathematical formulation in common (Section 3). The field equation, the Laplace Equation 3, is common. The boundary constraints are common, specifically the bottom boundary condition (Eq. 5), the kinematic free-surface boundary condition (Eq. 6), the dynamic free surface boundary condition (Eq. 7), the periodic lateral boundary conditions (Eq. 8), the wave height definition (Eq. 9), and an invariant mean water level (Eq. 10).

Each theory adopts a series solution of the stream function $\Psi$, Eq. 18 for the Stokes theory and Eq. 44 for the cnoidal theory, that exactly satisfies the field equation and the bottom boundary condition. The balance of the unknowns are represented as small parameter expansions in parameter $\varepsilon$ that depends on the wave height $H$. At zeroth order, the algebraic coefficients in these expansions correspond to uniform and critical flow. At 
higher order, these algebraic coefficients are uniquely determined from the balance of the boundary constraints.

A crucially important common feature is the dispersion relationship (Section 4) which relates the flow in the steady frame to the wave period $T$ and current $\left(U_{E}\right.$ or $\left.U_{S}\right)$ in the fixed frame. Separate Stokes first (Eq. 15) and second (Eq. 16) definitions of phase speed $C$ are identified.

A new Stokes theory is introduced in Section 5. The coefficients to fifth order are listed (Appendix A), together with a numerical list of coefficients (Appendix B) for a specified deep water wave condition. The theory is confirmed as correct to fifth order through an application of Richardson extrapolation to the limit.

The stream function $\Psi$ and water surface elevation $\eta$ are directly predicted by the Stokes theory. Predictive equations are given for the velocity components $(u, w)$, the acceleration components $(D u / D t, D w / D t)$ and the dynamic pressure $p_{d}$ in the fixed frame.

The limits of validity of the Stokes theory are explored (Figure 3 ) in dimensionless parameter space identified by the relative water depth $\omega^{2} h / g$ and by the relative wave height $H / H_{\text {Limit }}$ at that water depth. The extent of validity is clearly identified, extending from deep water to moderately shallow transitional depths, and up to relatively extreme wave heights that approach the limit wave. Examples of satisfactory (Figure 2) and unsatisfactory (Figure 4) application are given.

A new cnoidal theory is introduced in Section 6. The coefficients to fifth order are listed (Appendix C), together with a numerical list of coefficients (Appendix D) for a specified shallow water wave condition. The theory is confirmed as correct to fifth order through an application of Richardson extrapolation to the limit.

The water surface elevation $\eta$ is directly predicted by the cnoidal theory. But the balance of the kinematics require first and second derivatives of Jacobian elliptic functions. These are established exactly through a sequence of transformation matrices. Predicted equations are given for the stream function $\Psi$, the velocity components $(u, w)$, the acceleration components $(D u / D t, D w / D t)$ and the dynamic pressure $p_{d}$ in the fixed frame.

The limits of validity of the cnoidal theory are explored (Figure 6) in the same $\left(\omega^{2} h / g\right.$, $\left.H / H_{\text {Limit }}\right)$ parameter space. The extent of validity is clearly identified, extending from shallow water to moderately deep transitional depths, and up to moderately extreme wave heights. Examples of satisfactory (Figure 5) and unsatisfactory (Figure 7) application are again given.

Finally, the region of overlapping applicability of the Stokes and cnoidal theories is explored (Figure 8). It is suggested that $\omega^{2} h / g \approx 0.65$ separates the regions of optimal applicability for the cnoidal theory, $\omega^{2} h / g \lesssim 0.65$, and for the Stokes theory, $\omega^{2} h / g \gtrsim 0.65$. In the neighbourhood of this separation, the Stokes and cnoidal theories have comparable credibility (Figure 9). 


\section{A Stokes Coefficients to Fifth Order}

Non-zero $A_{i j}, B_{i j}, C_{i}, D_{i}$ and $E_{i}$ coefficients

[as indexed arrays $A(i, j), B(i, j), C(i), D(i)$ and $E(i)]$.

$q=\tanh (k h)$

$C(0)=\frac{1}{2} q, D(0)=q^{1 / 2}, E(0)=k h q^{1 / 2}$

$A(1,1)=1 / q^{1 / 2}, B(1,1)=1$

$C(1)=0, D(1)=0, E(1)=0$

$A(2,1)=0, A(2,2)=-3 / 8\left(q^{4}-1\right) / q^{7 / 2}$

$B(2,2)=-1 / 4\left(q^{2}-3\right) / q^{3}, B(2,1)=0$

$C(2)=1 / 16\left(5 q^{4}-6 q^{2}+9\right) / q^{3}$,

$D(2)=1 / 16 / q^{7 / 2}\left(9 q^{4}-10 q^{2}+9\right)$,

$E(2)=1 / 16\left(9 k h q^{4}-10 k h q^{2}+9 k h-8 q^{3}\right) / q^{7 / 2}$

$A(3,1)=1 / 64\left(39 q^{6}-41 q^{4}-3 q^{2}-27\right) / q^{13 / 2}, A(3,2)=0$

$A(3,3)=1 / 64\left(39 q^{6}-53 q^{4}+5 q^{2}+9\right) / q^{13 / 2}$

$B(3,1)=3 / 64\left(q^{6}-3 q^{4}+3 q^{2}-9\right) / q^{6}, B(3,2)=0$

$B(3,3)=-3 / 64\left(q^{6}-3 q^{4}+3 q^{2}-9\right) / q^{6}$

$C(3)=0, D(3)=0, E(3)=0$

$A(4,1)=0$,

$A(4,2)=-1 / 384\left(103 q^{10}+84 q^{8}-280 q^{6}-342 q^{4}+81 q^{2}+162\right) / q^{19 / 2}$,

$A(4,3)=0$,

$A(4,4)=-1 / 1536\left(197 q^{12}+1732 q^{10}+1481 q^{8}-9872 q^{6}+7623 q^{4}-756 q^{2}-405\right) / q^{19 / 2} /\left(q^{2}+5\right)$

$B(4,1)=0$,

$B(4,2)=1 / 96\left(3 q^{8}-88 q^{6}+198 q^{4}-81\right) / q^{9}$,

$B(4,3)=0$,

$B(4,4)=1 / 384\left(21 q^{10}+q^{8}-262 q^{6}+522 q^{4}+81 q^{2}+405\right) /\left(q^{2}+5\right) / q^{9}$

$C(4)=1 / 1024\left(33 q^{10}+55 q^{8}-1110 q^{6}+1710 q^{4}-27 q^{2}-405\right) / q^{9}$

$D(4)=1 / 1024\left(39 q^{10}+351 q^{8}-2194 q^{6}+2454 q^{4}-117 q^{2}-405\right) / q^{19 / 2}$

$E(4)=1 / 1024\left(39 k h q^{10}+351 k h q^{8}-2194 k h q^{6}+2454 k h q^{4}-117 k h q^{2}-405 k h-48 q^{9}+\right.$ $\left.112 q^{7}+48 q^{5}+144 q^{3}\right) / q^{19 / 2}$

$A(5,1)=1 / 4096\left(861 q^{16}+12262 q^{14}+30114 q^{12}-75986 q^{10}-131460 q^{8}-91438 q^{6}-199602 q^{4}+\right.$ $\left.89370 q^{2}+62775\right) /\left(3 q^{4}+20 q^{2}+25\right) / q^{25 / 2}$

$A(5,2)=0$

$A(5,3)=1 / 1024\left(519 q^{14}+2855 q^{12}-2157 q^{10}-11637 q^{8}+5613 q^{6}+8397 q^{4}-1863 q^{2}-\right.$ $1215) /\left(q^{2}+5\right) / q^{25 / 2}$

$A(5,4)=0$ 
$A(5,5)=1 / 4096\left(5415 q^{16}+32830 q^{14}-2142 q^{12}-121450 q^{10}+28240 q^{8}+135290 q^{6}-88578 q^{4}+\right.$ $\left.8370 q^{2}+2025\right) /\left(q^{2}+5\right) / q^{25 / 2} /\left(3 q^{2}+5\right)$

$B(5,1)=-1 / 12288\left(225 q^{16}-2370 q^{14}-14622 q^{12}+6070 q^{10}-53932 q^{8}+340410 q^{6}+764046 q^{4}-\right.$ $\left.203310 q^{2}-188325\right) /\left(3 q^{4}+20 q^{2}+25\right) / q^{12}$

$B(5,2)=0$

$B(5,3)=-9 / 1024\left(5 q^{12}+28 q^{10}-25 q^{8}+148 q^{6}-1305 q^{4}+216 q^{2}+405\right) /\left(q^{2}+5\right) / q^{12}$

$B(5,4)=0$

$B(5,5)=5 / 12288\left(45 q^{16}-150 q^{14}-570 q^{12}+2618 q^{10}-3896 q^{8}-498 q^{6}+25866 q^{4}+8910 q^{2}+\right.$ $6075) /\left(3 q^{4}+20 q^{2}+25\right) / q^{12}$

$C(5)=0, D(5)=0, E(5)=0$

\section{B Specific List of Stokes Coefficients}

$g=9.81 \mathrm{~m} / \mathrm{s}^{2}, h=100 \mathrm{~m}, H=10 \mathrm{~m}, T=10 \mathrm{~s}, U_{E}=0$

$k=0.03879 \mathrm{~m}^{-1}, \varepsilon=0.19394, L=161.99 \mathrm{~m} ; \omega^{2} h / g=4.020, \mathbf{U r}=0.262$

Non-zero $A_{i j}, B_{i j}, C_{i}, D_{i}$ and $E_{i}$ coefficients:

$\mathrm{C}(0)=0.49957, \mathrm{D}(0)=0.99957, \mathrm{E}(0)=3.87715$

$\mathrm{A}(1,1)=1.00043, \mathrm{~B}(1,1)=1.00000$

$\mathrm{A}(2,2)=0.00128, \mathrm{~B}(2,2)=0.50171$,

$\mathrm{C}(2)=0.50086, \mathrm{D}(2)=0.50064, \mathrm{E}(2)=1.44169$

$\mathrm{A}(3,1)=-0.50364, \mathrm{~A}(3,3)=-0.00043, \mathrm{~B}(3,1)=-0.37693, \mathrm{~B}(3,3)=0.37693$

$\mathrm{A}(4,2)=0.50142, \mathrm{~A}(4,4)=0.00012, \mathrm{~B}(4,2)=0.33332, \mathrm{~B}(4,4)=0.33567$,

$\mathrm{C}(4)=0.25118, \mathrm{D}(4)=0.12634, \mathrm{E}(4)=0.74122$

$\mathrm{A}(5,1)=-1.54900, \mathrm{~A}(5,3)=0.08560, \mathrm{~A}(5,5)=-0.00003$,

$\mathrm{B}(5,1)=-1.10530, \mathrm{~B}(5,3)=0.77688, \mathrm{~B}(5,5)=0.32841$

\section{Cnoidal Coefficients to Fifth Order}

$A_{i j}, B_{i j}, C_{i}, D_{i}, E_{i}, F_{i}$ coefficients [as indexed arrays $A(i, j), B(i, j), C(i), D(i), E(i)$ and $F(i)$ ].

$e=E(m) / K(m)$ 
$C(0)=0.5, D(0)=1, E(0)=1, F(0)=0$

$A(1,0)=1 / 2(-m+e) / m, A(1,1)=1, B(1,0)=(1-m-e) / m, B(1,1)=1$

$C(1)=1 / 2(2-m-3 e) / m, D(1)=1 / 2(2-m-3 e) / m$

$E(1)=1 / 2(2-m-3 e) / m, F(1)=3 / 4 / m$

$A(2,0)=1 / 40\left(-10 e-5 m e+5 e^{2}+34 m-24 m^{2}-4\right) / m^{2}$

$A(2,1)=1 / 4(-6+7 m+2 e) / m, A(2,2)=-1$

$B(2,0)=1 / 4(2 m+2 e-2-m e) / m^{2}, B(2,1)=-3 / 4, B(2,2)=3 / 4$

$C(2)=1 / 40\left(30 e-15 m e+16 m-m^{2}-16\right) / m^{2}$

$D(2)=1 / 120\left(190 e-95 m e-18 m^{2}+88 m-88-75 e^{2}\right) / m^{2}$

$E(2)=1 / 40\left(10 e-5 m e+15 e^{2}+16 m-16-6 m^{2}\right) / m^{2}$

$F(2)=1 / 16 / m^{2}(-30+15 m+36 e)$

$A(3,0)=1 / 2800\left(511 e+2989 m e+4997 m^{2}-4411 m-2639 m^{2} e-1320 m^{3}+584-1050 e^{2}+\right.$ $\left.175 e^{3}\right) / m^{3}$

$A(3,1)=1 / 40\left(-100 e+110 m e+109 m^{2}-229 m+101+15 e^{2}\right) / m^{2}$

$A(3,2)=1 / 10(32-34 m-15 e) / m, A(3,3)=6 / 5$

$B(3,0)=1 / 400\left(-399 m+133 m^{2}+466 m e-466 e+200 e^{2}-16 m^{2} e-100 m e^{2}+266\right) / m^{3}$

$B(3,1)=1 / 80(-1+51 m-60 e) / m$

$B(3,2)=1 / 80 / m(1-152 m+60 e), B(3,3)=101 / 80$

$C(3)=1 / 2800\left(-3913 e+3913 m e+519 m^{2}-1917 m-238 m^{2} e+60 m^{3}-1050 m e^{2}+1278+\right.$ $\left.2100 e^{2}\right) / m^{3}$

$D(3)=1 / 8400\left(-22799 e+22799 m e+4357 m^{2}-10371 m-2849 m^{2} e-450 m^{3}+6914-\right.$ $\left.9450 m e^{2}+18900 e^{2}-3675 e^{3}\right) / m^{3}$

$E(3)=1 / 2800\left(-2653 e+2653 m e+1079 m^{2}-2337 m-203 m^{2} e+1558-350 m e^{2}+700 e^{2}+\right.$ $\left.175 e^{3}-150 m^{3}\right) / m^{3}$

$F(3)=1 / 32\left(135-135 m+36 m^{2}-276 e+138 m e+144 e^{2}\right) / m^{3}$

$A(4,0)=1 / 336000\left(-72880 e-1196040 m e-1759052 m^{2}+1167024 m+1580356 m^{2} e-\right.$ $426828 m^{3} e+959060 m^{3}-163260 m^{4}-185232+308700 m e^{2}+268800 e^{2}-157500 e^{3}-400575 m^{2} e^{2}+$ $\left.26250 m e^{3}+13125 e^{4}\right) / m^{4}$

$A(4,1)=1 / 84000\left(-398280+652680 e-1387680 m e-1184248 m^{2}+1243772 m+629055 m^{2} e+\right.$ $\left.308580 m^{3}+301875 m e^{2}-288750 e^{2}+26250 e^{3}\right) / m^{3}$

$A(4,2)=1 / 6000\left(-46168+99280 m-45955 m^{2}+52500 e-53250 m e-11250 e^{2}\right) / m^{2}$

$A(4,3)=1 / 750(-4426+4577 m+2250 e) / m, A(4,4)=-197 / 125$

$B(4,0)=1 / 6000\left(19240 e-28860 m e-14724 m^{2}+19520 m+13058 m^{2} e-1719 m^{3} e+4964 m^{3}+\right.$ $\left.12480 m e^{2}-12480 e^{2}+3000 e^{3}-855 m^{2} e^{2}-1500 m e^{3}-9760\right) / m^{4}$

$B(4,1)=1 / 24000\left(-9728+8400 e-24645 m^{2}+26100 m e-18000 e^{2}+1537 m\right) / m^{2}$

$B(4,2)=1 / 24000\left(9728-8400 e+68555 m^{2}-86700 m e+18000 e^{2}+14845 m\right) / m^{2}$

$B(4,3)=1 / 24000(-16382+60600 e-96011 m) / m, B(4,4)=17367 / 8000$ 
$C(4)=1 / 84000\left(298440 e-447660 m e-137323 m^{2}+179824 m+215268 m^{2} e-33024 m^{3} e+\right.$ $\left.47411 m^{3}-1440 m^{4}+232680 m e^{2}-232680 e^{2}+63000 e^{3}-20055 m^{2} e^{2}-31500 m e^{3}-89912\right) / m^{4}$ $D(4)=1 / 336000\left(-499728+1946160 e-2919240 m e-790932 m^{2}+999456 m+1322872 m^{2} e-\right.$ $174896 m^{3} e+291204 m^{3}-18540 m^{4}+2192120 m e^{2}-2192120 e^{2}+997500 e^{3}-262745 m^{2} e^{2}-$ $\left.498750 m e^{3}-118125 e^{4}\right) / m^{4}$

$E(4)=1 / 336000\left(1022160 e-1533240 m e-739132 m^{2}+896416 m+859752 m^{2} e-174336 m^{3} e+\right.$ $290924 m^{3}-18540 m^{4}+521640 m e^{2}-521640 e^{2}+73500 e^{3}-41265 m^{2} e^{2}-36750 m e^{3}+7875 e^{4}-$ $448208) / m^{4}$

$F(4)=1 / 28000\left(-280544+420816 m-216562 m^{2}+38145 m^{3}+737520 e-737520 m e-\right.$ $\left.682500 e^{2}+341250 m e^{2}+186270 m^{2} e+210000 e^{3}\right) / m^{4}$

$A(5,0)=1 / 258720000\left(37082287 e+2779624826 m e+4306471322 m^{2}-2452998375 m-\right.$ $5140825459 m^{2} e+2947665886 m^{3} e-3359149003 m^{3}+1181590352 m^{4}-517470448 m^{4} e-$ $114888060 m^{5}-1242664500 m e^{2}+433834224-583036300 e^{2}+461249250 e^{3}+2182993120 m^{2} e^{2}+$ $\left.104700750 m e^{3}-141487500 e^{4}-619238235 m^{3} e^{2}-352303875 m^{2} e^{3}+35371875 m e^{4}+7074375 e^{5}\right) / m^{5}$ $A(5,1)=1 / 2352000\left(24880233-51887080 e+152927236 m e+120473679 m^{2}-89726610 m-\right.$ $138431272 m^{2} e+34830474 m^{3} e-66555854 m^{3}+11176116 m^{4}-77596890 m e^{2}+38494890 e^{2}-$ $\left.10290000 e^{3}+33735765 m^{2} e^{2}+10290000 m e^{3}+643125 e^{4}\right) / m^{4}$

$A(5,2)=1 / 588000\left(9991536-31890660 m+30796990 m^{2}-8559467 m^{3}-18774154 e+\right.$ $\left.38314570 m e+9775500 e^{2}-9518250 m e^{2}-17047345 m^{2} e-1286250 e^{3}\right) / m^{3}$

$A(5,3)=1 / 147000\left(2817226-3256736 e+2764041 m^{2}+3250072 m e+771750 e^{2}-5900090 m\right) / m^{2}$ $A(5,4)=1 / 36750(385358-202713 e-394249 m) / m, A(5,5)=13438 / 6125$

$B(5,0)=1 / 23520000\left(-252700954 e+505401908 m e+277519372 m^{2}-286155385 m-376502602 m^{2} e+\right.$ $123801648 m^{3} e-130123673 m^{3}+24297532 m^{4}-4834544 m^{4} e-304201800 m e^{2}+114462154+$ $202801200 e^{2}-76322400 e^{3}+143242680 m^{2} e^{2}+76322400 m e^{3}+11760000 e^{4}-20921040 m^{3} e^{2}-$ $\left.7232400 m^{2} e^{3}-5880000 m e^{4}\right) / m^{5}$

$B(5,1)=1 / 1568000\left(1260819 m^{3}+2824245-2669128 e+73892 m e+1705200 e^{2}-2463253 m+\right.$ $\left.1295557 m^{2}-4377660 m^{2} e+2116800 m e^{2}-1176000 e^{3}\right) / m^{3}$

$B(5,2)=1 / 1568000 / m^{3}\left(-2824245+2669128 e+5116580 m e-1705200 e^{2}-1082976 m-\right.$ $\left.266581 m^{2}-7509922 m^{3}+11994220 m^{2} e-8055600 m e^{2}+1176000 e^{3}\right)$

$B(5,3)=1 / 1568000 / m^{2}\left(-5190472 e+3546229+14979104 m^{2}-17828356 m e+5938800 e^{2}+\right.$ $1488277 m)$

$B(5,4)=1 / 1568000 / m(-2517253-15389086 m+10211796 e), B(5,5)=1331817 / 313600$

$C(5)=1 / 258720000\left(-2906319141 e+5812638282 m e+1987483620 m^{2}-2049633275 m-\right.$ $4378504053 m^{2} e+1472184912 m^{3} e-931592155 m^{3}+165733780 m^{4}-90562296 m^{4} e+4077360 m^{5}-$ $4185650700 m e^{2}+2790433800 e^{2}-1168767600 e^{3}+2062049220 m^{2} e^{2}+1168767600 m e^{3}+$ $\left.194040000 e^{4}-333416160 m^{3} e^{2}-125802600 m^{2} e^{3}-97020000 m e^{4}+819853310\right) / m^{5}$

$D(5)=-1 / 776160000\left(12249145623 e-24498291246 m e-7710738940 m^{2}+7552779225 m+\right.$ $18327679909 m^{2} e-6078534286 m^{3} e+4013329185 m^{3}-865095020 m^{4}+406028788 m^{4} e+$ $15418620 m^{5}+23757772500 m e^{2}-15838515000 e^{2}+9921669450 e^{3}-10745013510 m^{2} e^{2}-$ $9921669450 m e^{3}-2900493750 e^{4}+1412878005 m^{3} e^{2}+1269708825 m^{2} e^{3}+1450246875 m e^{4}+$ 
$\left.233454375 e^{5}-3021111690\right) / m^{5}$

$E(5)=1 / 258720000\left(-2683379501 e+5366759002 m e+2547858820 m^{2}-2530159475 m-\right.$ $4186667023 m^{2} e+1503287522 m^{3} e-1291628755 m^{3}+272144700 m^{4}-102902316 m^{4} e-5139540 m^{5}-$ $2969851500 m e^{2}+1979901000 e^{2}-576864750 e^{3}+1618466850 m^{2} e^{2}+576864750 m e^{3}+50531250 e^{4}-$ $\left.314258175 m^{3} e^{2}-56392875 m^{2} e^{3}-25265625 m e^{4}+3031875 e^{5}+1012063790\right) / m^{5}$ $F(5)=1 / 3136000\left(-248719128 e+373078692 m e+124296437 m^{2}-164036162 m-189430332 m^{2} e+\right.$ $32535384 m^{3} e-42278356 m^{3}+5042184 m^{4}-302455440 m e^{2}+302455440 e^{2}-170520000 e^{3}+$ $\left.73576440 m^{2} e^{2}+85260000 m e^{3}+35280000 e^{4}+82018081\right) / m^{5}$

\section{Specific List of Cnoidal Coefficients}

$g=9.81 \mathrm{~m} / \mathrm{s}^{2}, h=2 \mathrm{~m}, H=0.5 \mathrm{~m}, T=10 \mathrm{~s}$

$m=1-0.0013618, \varepsilon=0.25, L=46.28 \mathrm{~m} ; \omega^{2} h / g=0.080, \mathbf{U r}=121.4$

$A_{i j}, B_{i j}, C_{i}, D_{i}, E_{i}$ and $F_{i}$ coefficients:

$\mathrm{C}(0)=0.5, \mathrm{D}(0)=1, \mathrm{E}(0)=1, \mathrm{~F}(0)=0$

$\mathrm{A}(1,0)=-0.39291, \mathrm{~A}(1,1)=1.00000$

$\mathrm{B}(1,0)=-0.21281, \mathrm{~B}(1,1)=1.00000$

$\mathrm{C}(1)=0.18010, \mathrm{D}(1)=0.18010, \mathrm{E}(1)=0.18010 \mathrm{~F}(1)=0.75102$

$\mathrm{A}(2,0)=0.07623, \mathrm{~A}(2,1)=0.35504, \mathrm{~A}(2,2)=-1.00000$

$\mathrm{B}(2,0)=0.05301, \mathrm{~B}(2,1)=-0.75000, \mathrm{~B}(2,2)=0.75000$

$\mathrm{C}(2)=0.05499, \mathrm{D}(2)=-0.00965, \mathrm{E}(2)=-0.10650 \mathrm{~F}(2)=-0.45879$

$\mathrm{A}(3,0)=-0.00492, \mathrm{~A}(3,1)=-0.40590, \mathrm{~A}(3,2)=-0.51690, \mathrm{~A}(3,3)=1.20000$

$\mathrm{B}(3,0)=0.00305, \mathrm{~B}(3,1)=0.46435, \mathrm{~B}(3,2)=-1.72685, \mathrm{~B}(3,3)=1.26250$

$\mathrm{C}(3)=-0.02254, \mathrm{D}(3)=0.02829, \mathrm{E}(3)=0.04466 \mathrm{~F}(3)=0.41160$

$\mathrm{A}(4,0)=0.00210, \mathrm{~A}(4,1)=-0.61786, \mathrm{~A}(4,2)=1.08417, \mathrm{~A}(4,3)=0.83580$

$\mathrm{A}(4,4)=-1.57600$

$\mathrm{B}(4,0)=0.05666, \mathrm{~B}(4,1)=-1.09561, \mathrm{~B}(4,2)=3.06792, \mathrm{~B}(4,3)=-4.14319$

$\mathrm{B}(4,4)=2.17088$

$\mathrm{C}(4)=0.05960, \mathrm{D}(4)=0.03388, \mathrm{E}(4)=0.05091 \mathrm{~F}(4)=-0.42788$

$\mathrm{A}(5,0)=0.02825, \mathrm{~A}(5,1)=-0.22786, \mathrm{~A}(5,2)=1.47658, \mathrm{~A}(5,3)=-1.94665$

$\mathrm{A}(5,4)=-1.40901, \mathrm{~A}(5,5)=2.19396$

$\mathrm{B}(5,0)=-0.00550, \mathrm{~B}(5,1)=1.01588, \mathrm{~B}(5,2)=-5.03541, \mathrm{~B}(5,3)=9.79988$

$\mathrm{B}(5,4)=-10.02722, \mathrm{~B}(5,5)=4.24687$

$\mathrm{C}(5)=-0.03595, \mathrm{D}(5)=-0.02101, \mathrm{E}(5)=-0.01143 \mathrm{~F}(5)=0.22500$ 


\section{E Cnoidal Transformation Matrices}

(1) $d^{2} \mathrm{cn}^{2 j}(\chi) / d \chi^{2}$

Analytical differentiation leads to powers and products of Jacobian $\operatorname{sn}(\chi), \operatorname{cn}(\chi)$ and $\operatorname{dn}(\chi)$ terms. These powers and products are transformed to sums of powers of $\operatorname{cn}^{2}(\chi)$, using identities among these functions based on $\operatorname{sn}^{2}(\chi)=1-\operatorname{cn}^{2}(\chi)$ and $\operatorname{dn}^{2}(\chi)=1-m+m \mathrm{cn}^{2}(\chi)$ [Gradshteyn \& Ryzhik $(1965, \S 8.154(4,5))$ ]. The generic result is

$$
\begin{aligned}
\frac{d^{2}}{d \chi^{2}} \mathrm{cn}^{2 j}(\chi)=2 j(2 j-1)(1-m) \mathrm{cn}^{2 j-2}(\chi)+4 j^{2}(2 m-1) & \mathrm{cn}^{2 j}(\chi) \\
& -2 j(2 j+1) m \mathrm{cn}^{2 j+2}(\chi)
\end{aligned}
$$

The equivalent transformation matrix is

$$
\mathbb{F}=\left[\begin{array}{cccccc}
0 & 0 & 0 & 0 & 0 & 0 \\
2-2 m & 8 m-4 & -6 m & 0 & 0 & 0 \\
0 & 12-12 m & 32 m-16 & -20 m & 0 & 0 \\
0 & 0 & 30-30 m & 72 m-36 & -42 m & 0 \\
0 & 0 & 0 & 56-56 m & 128 m-64 & -72 m \\
0 & 0 & 0 & 0 & 90-90 m & 200 m-100
\end{array}\right]
$$

(2) $d \operatorname{cn}^{2 j}(\chi) / d \chi$

In a similar manner,

$$
\frac{d}{d \chi} \operatorname{cn}^{2 j}(\chi)=-2 j \operatorname{scd}(\chi) \operatorname{cn}^{2 j-2}(\chi)
$$

The equivalent transformation matrix is

$$
\mathbb{G}_{0}=\left[\begin{array}{cccccc}
0 & 0 & 0 & 0 & 0 & 0 \\
-2 & 0 & 0 & 0 & 0 & 0 \\
0 & -4 & 0 & 0 & 0 & 0 \\
0 & 0 & -6 & 0 & 0 & 0 \\
0 & 0 & 0 & -8 & 0 & 0 \\
0 & 0 & 0 & 0 & -10 & 0
\end{array}\right]
$$

(3) $d^{2} \operatorname{scd}(\chi) \operatorname{cn}^{2 j}(\chi) / d \chi^{2}$ 
In a similar manner again,

$$
\begin{aligned}
\frac{d^{2}}{d \chi^{2}} \operatorname{scd}(\chi) \operatorname{cn}^{2 j}(\chi)=\operatorname{scd}(\chi) & {\left[2(j-1)(2 j-1)(1-m) \operatorname{cn}^{2 j-4}(\chi)\right.} \\
+ & \left.4 j^{2}(2 m-1) \operatorname{cn}^{2 j-2}(\chi)-2(j+1)(2 j+1) m \operatorname{cn}^{2 j}(\chi)\right]
\end{aligned}
$$

The equivalent transformation matrix is

$$
\mathbb{G}=\left[\begin{array}{cccccc}
8 m-4 & -12 m & 0 & 0 & 0 & 0 \\
6-6 m & 32 m-16 & -30 m & 0 & 0 & 0 \\
0 & 20-20 m & 72 m-36 & -56 m & 0 & 0 \\
0 & 0 & 42-42 m & 128 m-64 & -90 m & 0 \\
0 & 0 & 0 & 72-72 m & 200 m-100 & -132 m \\
0 & 0 & 0 & 0 & 110-110 m & 288 m-144
\end{array}\right]
$$

(4) $\left[F_{i}\right]$

Multiplication by $\alpha^{2}$ in the definition of $f_{k}(\chi)$ (Eq. 58) is equivalent to the transformation matrix

$$
\left[F_{i}\right]=\left[\begin{array}{cccccc}
F_{0} & 0 & 0 & 0 & 0 & 0 \\
F_{1} & F_{0} & 0 & 0 & 0 & 0 \\
F_{2} & F_{1} & F_{0} & 0 & 0 & 0 \\
F_{3} & F_{2} & F_{1} & F_{0} & 0 & 0 \\
F_{4} & F_{3} & F_{2} & F_{1} & F_{0} & 0 \\
F_{5} & F_{4} & F_{3} & F_{2} & F_{1} & F_{0}
\end{array}\right]
$$

\section{References}

J. D. Fenton (1979). 'A high-order cnoidal wave theory'. Journal of Fluid Mechanics 94:129-161.

J. D. Fenton (1985). 'A fifth-order Stokes theory for steady waves'. Journal of Waterway, Port, Coastal and Ocean Engineering, ASCE 111:216-234. Errata, 113, p.438.

I. S. Gradshteyn \& I. M. Ryzhik (1965). Table of Integrals, Series and Products. Academic Press, New York.

M. Isobe \& N. C. Kraus (1983). 'Derivation of a third-order Stokes wave theory'. Technical Report YNU-HY-83-1, Department of Civil Engineering, Yokohama National University, Hydraulics Laboratory, Yokohama, Japan.

M. Isobe, et al. (1982). 'Theoretical considerations on perturbation solutions for waves of permanent type'. Bulletin of Faculty of Engineering, Yokohama National University 31:29-57. 
J. B. Keller (1948). 'The solitary wave and periodic waves in shallow water'. Communications on Pure and Applied Mathematics 1:323-339.

N. Kishida \& R. J. Sobey (1988). 'Stokes theory for waves on linear shear current'. Journal of Engineering Mechanics 114:1317-1334.

E. V. Laitone (1960). 'The second approximation to cnoidal and solitary waves'. Journal of Fluid Mechanics 9:430-444.

H. Lamb (1932). Hydrodynamics. Cambridge University Press, Cambridge, 6 edn.

H. Nishimura, et al. (1977). 'Higher order solutions of the Stokes and cnoidal waves'. Journal of the Faculty of Engineering, University of Tokyo, Series B 34:267-293.

L. W. Schwartz (1974). 'Computer extension and analytical continuation of Stokes' expansion for gravity waves'. Journal of Fluid Mechanics 65:553-578.

R. J. Sobey (1989). 'Variations on Fourier wave theory'. International Journal for Numerical Methods in Fluids 9:1453-1467.

R. J. Sobey (2009). 'Analytical Solutions for Steep Standing Waves'. Engineering and Computational Mechanics 162(EM4):185-197.

G. G. Stokes (1880). Mathematical and Physical Papers, Volume I. University Press, Cambridge.

J. M. Williams (1985). Tables of Progressive Gravity Waves. Pitman, Boston. 